\title{
Some results of algebraic geometry over Henselian rank one valued fields
}

\author{
Krzysztof Jan Nowak ${ }^{1}$
}

Published online: 13 June 2016

(C) The Author(s) 2016. This article is published with open access at Springerlink.com

\begin{abstract}
We develop geometry of affine algebraic varieties in $K^{n}$ over Henselian rank one valued fields $K$ of equicharacteristic zero. Several results are provided including: the projection $K^{n} \times \mathbb{P}^{m}(K) \rightarrow K^{n}$ and blowups of the $K$-rational points of smooth $K$-varieties are definably closed maps; a descent property for blowups; curve selection for definable sets; a general version of the Łojasiewicz inequality for continuous definable functions on subsets locally closed in the $K$-topology; and extending continuous hereditarily rational functions, established for the real and $p$-adic varieties in our joint paper with J. Kollár. The descent property enables application of resolution of singularities and transformation to a normal crossing by blowing up in much the same way as over the locally compact ground field. Our approach relies on quantifier elimination due to Pas and a concept of fiber shrinking for definable sets, which is a relaxed version of curve selection. The last three sections are devoted to the theory of regulous functions and sets over such valued fields. Regulous geometry over the real ground field $\mathbb{R}$ was developed by Fichou-Huisman-Mangolte-Monnier. The main results here are regulous versions of Nullstellensatz and Cartan's theorems A and B.
\end{abstract}

Keywords Closedness theorem - Descent property for blowups · Curve selection · Łojasiewicz inequality - Hereditarily rational functions - Regulous functions and sets · Nullstellensatz · Cartan's theorems A and B

Mathematics Subject Classification Primary 12J25 • 03C10; Secondary 14G27 . $14 \mathrm{P} 10$

Krzysztof Jan Nowak nowak@im.uj.edu.pl

1 Institute of Mathematics, Faculty of Mathematics and Computer Science, Jagiellonian University, ul. Profesora Łojasiewicza 6, 30-348 Kraków, Poland 


\section{Introduction}

In this paper, we develop geometry of affine algebraic varieties in $K^{n}$ over Henselian rank one valued fields $K$ of equicharacteristic zero with valuation $v$, value group $\Gamma$, valuation ring $R$ and residue field $\mathbb{k}$. Every rank one valued field has a metric topology induced by its absolute value. Examples of such fields are the quotient fields of the rings of formal power series and of Puiseux series with coefficients from a field $\mathbb{k}$ of characteristic zero as well as the fields of Hahn series (maximally complete valued fields also called Malcev-Neumann fields; cf. [24]):

$$
\mathbb{k}\left(\left(t^{\Gamma}\right)\right):=\left\{f(t)=\sum_{\gamma \in \Gamma} a_{\gamma} t^{\gamma}: a_{\gamma} \in \mathbb{k}, \quad \text { supp } f(t) \text { is well ordered }\right\} .
$$

Let $X$ be a $K$-algebraic variety. We always assume that $X$ is reduced, but we allow it to be reducible. The set $X(K)$ of its $K$-rational points ( $K$-points for short) inherits from $K$ a topology, called the $K$-topology. In this paper, we are going to investigate continuous and differentiable functions $X(K) \rightarrow K$ that come from algebraic geometry and their zero sets. Therefore, we shall (and may) most often assume that $X$ is an affine $K$-variety such that $X(K)$ is Zariski dense in $X$. Throughout the paper, by "definable" we shall mean "definable with parameters."

Several results concerning algebraic geometry over such ground fields $K$ are established. Let $\mathcal{L}$ be the three-sorted language of Denef-Pas. We prove that the projection

$$
K^{n} \times \mathbb{P}^{m}(K) \rightarrow K^{n}
$$

is an $\mathcal{L}$-definably closed map (Theorem 3.1 ). Further, we shall draw several conclusions, including the theorem that blowups of the $K$-points of smooth $K$-varieties are definably closed maps (Corollary 3.5), a descent property for such blowups (Corollary 3.6), curve selection for $\mathcal{L}$-definable sets (Proposition 8.2 ) and for valuative semialgebraic sets (Proposition 8.1) as well as a general version of the Łojasiewicz inequality for continuous $\mathcal{L}$-definable functions on subsets locally closed in the $K$-topology (Proposition 9.2). Also given is a theorem on extending continuous hereditarily rational functions over such ground fields (Theorem 10.2), established for the real and $p$-adic varieties in our joint paper [27] with J. Kollár. The proof makes use of the descent property and the Łojasiewicz inequality. The descent property enables application of resolution of singularities and transformation to a normal crossing by blowing up (see [25, Chap. III] for references and relatively short proofs) in much the same way as over the locally compact ground field. Our approach relies on quantifier elimination due to Pas and on a certain concept of fiber shrinking for definable sets, which is a relaxed version of curve selection. Note that this paper comprises our two earlier preprints $[39,40]$.

Remark 1.1 This paper is principally devoted to geometry over rank one valued fields (in other words, fields with non-archimedean absolute value). Therefore, from Sect. 3 on, we shall most often assume that so is the ground field $K$. Nevertheless, it is plausible that the closedness theorem (Theorem 2.6) and curve selection (Propositions 8.1 and 8.2 ) hold over arbitrary Henselian valued fields. 
We should emphasize that our approach to the subject of this paper is possible just because the language $\mathcal{L}$ in which we investigate valued fields is not too rich; in particular, it does not contain the inclusion language on the auxiliary sorts and the only symbols of $\mathcal{L}$ connecting the sorts are two functions from the main $K$-sort to the auxiliary $\Gamma$-sort and $\mathbb{k}$-sort. Hence and by elimination of $K$-quantifiers, the $\mathcal{L}$ definable subsets of the products of the two auxiliary sorts are precisely finite unions of the Cartesian products of sets definable in those two sorts. This allows us to reduce our reasonings to an analysis of ordinary cells (i.e., fibers of a cell in the sense of Pas).

The organization of the paper is as follows. In Sect. 2, we set up notation and terminology including, in particular, the language $\mathcal{L}$ of Denef-Pas and the concept of a cell. We recall the theorems on quantifier elimination and on preparation cell decomposition, due to Pas [41]. Next we draw some conclusions as, for instance, Corollary 2.3 on definable functions and Corollary 2.7 on certain decompositions of definable sets. The former will be applied in Sect. 5, and the latter is crucial for our proof of the closedness theorem (Theorem 3.1), which is stated in Sect. 3 together with several direct corollaries, including the descent property. Section 4 gives a proof (being of algorithmic character) of this theorem for the case where the value group $\Gamma$ is discrete.

In Sect. 5, we study $\mathcal{L}$-definable functions of one variable. A result playing an important role in the sequel is the theorem on existence of the limit (Proposition 5.2). Its proof makes use of Puiseux's theorem for the local ring of convergent power series. In Sect. 6, we introduce a certain concept of fiber shrinking for $\mathcal{L}$-definable sets (Proposition 6.1), which is a relaxed version of curve selection. Section 7 provides a proof of the closedness theorem (Theorem 3.1) for the general case. This proof makes use of fiber shrinking and existence of the limit for functions of one variable.

In the subsequent three sections, some further conclusions from the closedness theorems are drawn. Section 8 provides some versions of curve selection: for arbitrary $\mathcal{L}$-definable sets and for valuative semialgebraic sets. The next section is devoted to a general version of the Łojasiewicz inequality for continuous $\mathcal{L}$-definable functions on subsets locally closed in the $K$-topology (Proposition 9.2). In Sect. 10, the theorem on extending continuous hereditarily rational functions (established for the real and $p$ adic varieties in [27]) is carried over to the case where the ground field $K$ is a Henseliam rank one valued field of equicharacteristic zero. Let us mention that in real algebraic geometry applications of continuous hereditarily rational functions and the extension theorem, in particular, are given in the papers [28-30] and [31], which discuss rational maps into spheres and stratified-algebraic vector bundles on real algebraic varieties.

The last three sections are devoted to the theory of regulous functions and sets over Henselian rank one valued fields of equicharacteristic zero. Regulous geometry over the real ground field $\mathbb{R}$ was developed by Fichou-Huisman-Mangolte-Monnier [16]. In Sect. 11, we set up notation and terminology as well as provide basic results about regulous functions and sets, including the noetherianity of the constructible and regulous topologies. Those results are valid over arbitrary fields with the density property. The next section establishes a regulous version of Nullstellensatz (Theorem 12.4), valid over Henselian rank one valued fields of equicharacteristic zero. The proof relies on the Łojasiewicz inequality (Proposition 9.2). Also drawn are several conclusions, including the existence of a one-to-one correspondence between the radical ideals of 
the ring of regulous functions and the closed regulous subsets, or one-to-one correspondences between the prime ideals of that ring, the irreducible regulous subsets and the irreducible Zariski closed subsets (Corollaries 12.5 and 12.10).

Section 13 provides an exposition of the theory of quasi-coherent regulous sheaves, which generally follows the approach given in the real algebraic case by FichouHuisman-Mangolte-Monnier [16]. It is based on the equivalence of categories between the category of $\widetilde{\mathcal{R}}^{k}$-modules on the affine scheme $\operatorname{Spec}\left(\mathcal{R}^{k}\left(K^{n}\right)\right)$ and the category of $\mathcal{R}^{k}$-modules on $K^{n}$ which, in turn, is a direct consequence of the one-to-one correspondences mentioned above. The main results here are the regulous versions of Cartan's theorems A and B. We also establish a criterion for a continuous function on an affine regulous subvariety to be regulous (Proposition 13.10), which relies on our theorem on extending continuous hereditarily rational functions (Theorem 10.2).

Note finally that the metric topology of a non-archimedean field $K$ with a rank one valuation $v$ is totally disconnected. Rigid analytic geometry (see, e.g., [6] for its comprehensive foundations), developed by Tate, compensates for this defect by introducing sheaves of analytic functions in a Grothendieck topology. Another approach is due to Berkovich [3], who filled in the gaps between the points of $K^{n}$, producing a locally compact Hausdorff space (the analytification of $K^{n}$ ), which contains the metric space $K^{n}$ as a dense subspace whenever the ground field $K$ is algebraically closed. His construction consists in replacing each point $x$ of a given $K$-variety with the space of all rank one valuations on the residue field $\kappa(x)$ that extend $v$. Further, the theory of stably dominated types, developed by Hrushovski-Loeser [23], deals with non-archimedean fields with valuation of arbitrary rank and generalizes that of tame topology for Berkovich spaces. Currently, various analytic structures over Henselian rank one valued fields are intensively investigated (see, e.g., [11,12] for more information and [34] for the case of algebraically closed valued fields).

\section{Quantifier elimination and cell decomposition}

We begin with quantifier elimination due to Pas in the language $\mathcal{L}$ of Denef-Pas with three sorts: the valued field $K$-sort, the value group $\Gamma$-sort and the residue field $\mathbb{k}$ sort. The language of the $K$-sort is the language of rings; that of the $\Gamma$-sort is any augmentation of the language of ordered abelian groups (and $\infty$ ); finally, that of the $\mathbb{k}$-sort is any augmentation of the language of rings. We denote $K$-sort variables by $x, y, z, \ldots, \mathbb{k}$-sort variables by $\xi, \zeta, \eta, \ldots$, and $\Gamma$-sort variables by $k, q, r, \ldots$

In the case of non-algebraically closed fields, passing to the three sorts with additional two maps: the valuation $v$ and the residue map, is not sufficient. Quantifier elimination due to Pas holds for Henselian valued fields of equicharacteristic zero in the above three-sorted language with additional two maps: the valuation map $v$ from the field sort to the value group and a map $\overline{a c}$ from the field sort to the residue field (angular component map) which is multiplicative, sends 0 to 0 and coincides with the residue map on units of the valuation ring $R$ of $K$.

Not all valued fields $K$ have an angular component map, but it exists if $K$ has a cross section, which happens whenever $K$ is $\aleph_{1}$-saturated (cf. [9, Chap. II]). Moreover, a valued field $K$ has an angular component map whenever its residue field $\mathbb{k}$ 
is $\aleph_{1}$-saturated (cf. [42, Corollary 1.6]). In general, unlike for $p$-adic fields and their finite extensions, adding an angular component map does strengthen the family of definable sets. For both $p$-adic fields (Denef [13]) and Henselian equicharacteristic zero valued fields (Pas [41]), quantifier elimination was established by means of cell decomposition and a certain preparation theorem (for polynomials in one variable with definable coefficients) combined with each other. In the latter case, however, cells are no longer finite in number, but parametrized by residue field variables. In the proof of the closedness theorem, which is a fundamental tool for many results of this paper, we may use an angular component map because a given valued field can always be replaced with an $\aleph_{1}$-saturated elementary extension.

Finally, let us mention that quantifier elimination based on the sort $R V:=K^{*} /(1+$ $\mathfrak{m}) \cup\{0\}$ (where $K^{*}:=K \backslash\{0\}$ and $\mathfrak{m}$ is the maximal ideal of the valuation ring $R$ ) was introduced by Besarab [4]. This new sort binds together the value group and residue field into one structure. In the paper [22, Sect. 12], quantifier elimination for Henselian valued fields of equicharacteristic zero, based on this sort, was derived directly from that by Robinson [43] for algebraically closed valued fields. Yet another, more general result, including Henselian valued fields of mixed characteristic, was achieved by Cluckers-Loeser [10] for so-called b-minimal structures (from "ball minimal"); in the case of valued fields, however, countably many sorts $R V_{n}:=K^{*} /(1+n \mathfrak{m}) \cup\{0\}$, $n \in \mathbb{N}$, are needed.

Below we state the theorem on quantifier elimination due to Pas [41, Theorem 4.1].

Theorem 2.1 Let $(K, \Gamma, \mathbb{k})$ be a structure for the three-sorted language $\mathcal{L}$ of DenefPas. Assume that the valued field $K$ is Henselian and of equicharacteristic zero. Then $(K, \Gamma, \mathbb{k})$ admits elimination of $K$-quantifiers in the language $\mathcal{L}$.

We immediately obtain the following

Corollary 2.2 The three-sorted structure $(K, \Gamma, \mathbb{k})$ admits full elimination of quantifiers whenever the theories of the value group $\Gamma$ and the residue field $\mathbb{k}$ admit quantifier elimination in the languages of their sorts.

Below we prove another consequence of elimination of $K$-quantifiers, which will be applied to the study of definable functions of one variable in Sect. 5 .

Corollary 2.3 Let $f: A \rightarrow K$ be an $\mathcal{L}$-definable function on a subset $A$ of $K^{n}$. Then there is a finite partition of $A$ into $\mathcal{L}$-definable sets $A_{i}$ and irreducible polynomials $P_{i}(x, y), i=1, \ldots, k$, such that for each $a \in A_{i}$ the polynomial $P_{i}(a, y)$ in $y$ does not vanish and

$$
P_{i}(a, f(a))=0 \text { for all } a \in A_{i}, \quad i=1, \ldots, k \text {. }
$$

Proof By elimination of $K$-quantifiers, the graph of $f$ is a finite union of sets $B_{i}$, $i=1, \ldots, k$, defined by conditions of the form

$$
\left(v\left(f_{1}(x, y)\right), \ldots, v\left(f_{r}(x, y)\right)\right) \in P, \quad\left(\overline{a c} g_{1}(x, y), \ldots, \overline{a c} g_{s}(x, y)\right) \in Q,
$$


where $f_{i}, g_{j} \in K[x, y]$ are polynomials, and $P$ and $Q$ are $\mathcal{L}$-definable subsets of $\Gamma^{r}$ and $\mathbb{K}^{s}$, respectively. Each set $B_{i}$ is the graph of the restriction of $f$ to an $\mathcal{L}$ definable subset $A_{i}$. Since, for each point $a \in A_{i}$, the fiber of $B_{i}$ over $a$ consists of one point, the above condition imposed on angular components includes one of the form $\overline{a c} g_{j}(x, y)=0$ or, equivalently, $g_{j}(x, y)=0$, for some $j=1, \ldots, s$, which may depend on $a$, where the polynomial $g_{j}(a, y)$ in $y$ does not vanish. This means that the set

$$
\left\{\left(\overline{a c} g_{1}(x, y), \ldots, \overline{a c} g_{s}(x, y)\right):(x, y) \in B_{i}\right\}
$$

is contained in the union of hyperplanes $\bigcup_{j=1}^{s}\left\{\xi_{j}=0\right\}$ and, furthermore, that for each point $a \in A_{i}$ there is an index $j=1, \ldots, s$ such that the polynomial $g_{j}(a, y)$ in $y$ does not vanish and $g_{j}(a, f(a))=0$. Clearly, for any $j=1, \ldots, s$, this property of points $a \in A_{i}$ is $\mathcal{L}$-definable. Therefore, we can partition the set $A_{i}$ into subsets each of which fulfills the condition required in the conclusion with some irreducible factors of the polynomial $g_{j}(x, y)$.

Recall now some notation concerning cell decomposition. Consider an $\mathcal{L}$-definable subset $D$ of $K^{n} \times \mathbb{k}^{m}$, three $\mathcal{L}$-definable functions

$$
a(x, \xi), b(x, \xi), c(x, \xi): D \rightarrow K
$$

and a positive integer $v$. For each $\xi \in \mathbb{k}^{m}$ set

$$
\begin{aligned}
& C(\xi):=\left\{(x, y) \in K_{x}^{n} \times K_{y}:(x, \xi) \in D,\right. \\
& \left.\quad v(a(x, \xi)) \triangleleft_{1} v\left((y-c(x, \xi))^{v}\right) \triangleleft_{2} v(b(x, \xi)), \overline{a c}(y-c(x, \xi))=\xi_{1}\right\},
\end{aligned}
$$

where $\triangleleft_{1}, \triangleleft_{2}$ stand for $<, \leq$ or no condition in any occurrence. If the sets $C(\xi)$, $\xi \in \mathbb{k}^{m}$, are pairwise disjoint, the union

$$
C:=\bigcup_{\xi \in \mathbb{k}^{m}} C(\xi)
$$

is called a cell in $K^{n} \times K$ with parameters $\xi$ and center $c(x, \xi) ; C(\xi)$ is called a fiber of the cell $C$.

Theorem 2.4 (Preparation Cell Decomposition, [41, Theorem 3.2]) Let

$$
f_{1}(x, y), \ldots, f_{r}(x, y)
$$

be polynomials in one variable $y$ with coefficients being $\mathcal{L}$-definable functions on $K_{x}^{n}$. Then $K^{n} \times K$ admits a finite partition into cells such that on each cell $C$ with parameters $\xi$ and center $c(x, \xi)$ and for all $i=1, \ldots, r$ we have:

$$
v\left(f_{i}(x, y)\right)=v\left(\tilde{f}_{i}(x, \xi)(y-c(x, \xi))^{v_{i}}\right), \overline{a c} f_{i}(x, y)=\xi_{\mu(i)},
$$


where $\tilde{f}_{i}(x, \xi)$ are $\mathcal{L}$-definable functions, $v_{i} \in \mathbb{N}$ for all $i=1, \ldots, r$, and the map $\mu:\{1, \ldots, r\} \rightarrow\{1, \ldots, m\}$ does not depend on $x, y, \xi$.

Remark 2.5 The functions $f_{1}(x, y), \ldots, f_{r}(x, y)$ are said to be prepared with respect to the variable $y$.

Every divisible ordered group $\Gamma$ admits quantifier elimination in the language $(<$ $,+,-, 0)$ of ordered groups. Therefore, it is not difficult to deduce from Theorems 2.1 and 2.4 the following

Corollary 2.6 (Cell decomposition) If, in addition, the value group $\Gamma$ is divisible, then every $\mathcal{L}$-definable subset $B$ of $K^{n} \times K$ is a finite disjoint union of cells.

Every archimedean ordered group $\Gamma$ (which of course may be regarded as a subgroup of the additive group $\mathbb{R}$ of real numbers) admits quantifier elimination in the Presburger language $(<,+,-, 0,1)$ with binary relation symbols $\equiv_{n}$ for congruences modulo $n>1, n \in \mathbb{N}$, where 1 denotes the minimal positive element of $\Gamma$ if it exists or $1=0$ otherwise. Under the circumstances, one can deduce in a similar manner the following

Corollary 2.7 If, in addition, the valuation $v$ is of rank 1 , then every $\mathcal{L}$-definable subset $B$ of $K^{n} \times K$ is a finite disjoint union of sets each of which is a subset

$$
F:=\bigcup_{\xi \in \mathbb{R}^{m}} F(\xi)
$$

of a cell

$$
C:=\bigcup_{\xi \in \mathbb{K}^{m}} C(\xi)
$$

determined by finitely many congruences:

$$
F(\xi)=\left\{(x, y) \in C(\xi): v\left(f_{i}(x, \xi)(y-c(x, \xi))^{k_{i}}\right) \equiv_{M} 0, \quad i=1, \ldots, s\right\}
$$

where $f_{i}$ are $\mathcal{L}$-definable functions, $k_{i} \in \mathbb{N}$ for $i=1, \ldots, s$, and $M \in \mathbb{N}, M>1$.

Remark 2.8 Corollary 2.7 will be applied to establish the closedness theorem (Theorem 3.1) in Sect. 7.

\section{Closedness theorem}

In this paper, we are interested mainly in geometry over a Henselian rank one valued field of equicharacteristic zero. From now on, we shall assume (unless otherwise stated) that the ground field $K$ is such a field. Below we state one of the basic theorems, on which many other results of our paper rely. 
Theorem 3.1 (Closedness theorem) Let $D$ be an $\mathcal{L}$-definable subset of $K^{n}$. Then the canonical projection

$$
\pi: D \times R^{m} \longrightarrow D
$$

is definably closed in the $K$-topology, i.e., if $B \subset D \times R^{m}$ is an $\mathcal{L}$-definable closed subset, so is its image $\pi(B) \subset D$.

Observe that the $K$-topology is $\mathcal{L}$-definable whence the above theorem is a firstorder property. Therefore, it can be proven using elementary extensions, and thus, one may assume that an angular component map exists. We shall provide two different proofs for this theorem. The first, given in Sect. 4, is valid whenever the value group $\Gamma$ is discrete, and is based on a procedure of algorithmic character. The other, given in Sect. 7, is valid for the general case and makes use of Corollary 2.7 and fiber shrinking from Sect. 6 which, in turn, relies on some results on $\mathcal{L}$-definable functions of one variable from Sect. 5 . When the ground field $K$ is locally compact, the closedness theorem holds by a routine topological argument. We immediately obtain five corollaries stated below.

Corollary 3.2 Let $D$ be an $\mathcal{L}$-definable subset of $K^{n}$ and $\mathbb{P}^{m}(K)$ stand for the projective space of dimension $m$ over $K$. Then the canonical projection

$$
\pi: D \times \mathbb{P}^{m}(K) \longrightarrow D
$$

is definably closed.

Corollary 3.3 Let $A$ be a closed $\mathcal{L}$-definable subset of $\mathbb{P}^{m}(K)$ or $R^{m}$. Then every continuous $\mathcal{L}$-definable map $f: A \rightarrow K^{n}$ is definably closed in the $K$-topology.

Corollary 3.4 Let $\phi_{i}, i=0, \ldots, m$, be regular functions on $K^{n}, D$ be an $\mathcal{L}$-definable subset of $K^{n}$ and $\sigma: Y \longrightarrow K \mathbb{A}^{n}$ the blowup of the affine space $K \mathbb{A}^{n}$ with respect to the ideal $\left(\phi_{0}, \ldots, \phi_{m}\right)$. Then the restriction

$$
\sigma: Y(K) \cap \sigma^{-1}(D) \longrightarrow D
$$

is a definably closed quotient map.

Proof Indeed, $Y(K)$ can be regarded as a closed algebraic subvariety of $K^{n} \times \mathbb{P}^{m}(K)$ and $\sigma$ as the canonical projection.

Since the problem is local with respect to the target space, the above corollary immediately generalizes to the case where the $K$-variety $Y$ is the blowup of a smooth $K$-variety $X$.

Corollary 3.5 Let $X$ be a smooth $K$-variety, $\phi_{i}, i=0, \ldots, m$, regular functions on $X, D$ be an $\mathcal{L}$-definable subset of $X(K)$ and $\sigma: Y \longrightarrow X$ the blowup of the ideal $\left(\phi_{0}, \ldots, \phi_{m}\right)$. Then the restriction

$$
\sigma: Y(K) \cap \sigma^{-1}(D) \longrightarrow D
$$


is a definably closed quotient map.

Corollary 3.6 (Descent property) Under the assumptions of the above corollary, every continuous $\mathcal{L}$-definable function

$$
g: Y(K) \cap \sigma^{-1}(D) \longrightarrow K
$$

that is constant on the fibers of the blowup $\sigma$ descends to a (unique) continuous $\mathcal{L}$-definable function $f: D \longrightarrow K$.

\section{Proof of Theorem 3.1 when the valuation is discrete}

The proof given in this section is of algorithmic character. Through the transfer principle of Ax-Kochen-Ershov (see, e.g., [9]), it suffices to prove Theorem 3.1 for the case where the ground field $K$ is a complete, discretely valued field of equicharacteristic zero. Such fields are, by virtue of Cohen's structure theorem, the quotient fields $K=\mathbb{k}((t))$ of formal power series rings $\mathbb{k}[[t]]$ in one variable $t$ with coefficients from a field $\mathbb{k}$ of characteristic zero. The valuation $v$ and the angular component $\overline{a c}$ of a formal power series are the degree and the coefficient of its initial monomial, respectively.

The additive group $\mathbb{Z}$ is an example of ordered $Z$-group, i.e., an ordered abelian group with a (unique) smallest positive element (denoted by 1 ) subject to the following additional axioms:

$$
\forall k k>0 \Rightarrow k \geq 1
$$

and

$$
\forall k \exists q \bigvee_{r=0}^{n-1} k=n q+r
$$

for all integers $n>1$. The language of the value group sort will be the Presburger language of ordered $Z$-groups, i.e., the language of ordered groups $\{<,+,-, 0\}$ augmented by 1 and binary relation symbols $\equiv_{n}$ for congruence modulo $n$ subject to the axioms:

$$
\forall k, r k \equiv_{n} r \Leftrightarrow \exists q k-r=n q
$$

for all integers $n>1$. This theory of ordered $Z$-groups has quantifier elimination and definable Skolem (choice) functions. We can replace the above two countable axiom schemas with universal ones after adding the unary function symbols $\left[\frac{k}{n}\right]$ of one variable $k$ for division by $n$ with remainder, which fulfill the following postulates:

$$
\left[\begin{array}{l}
k \\
n
\end{array}\right]=q \Leftrightarrow \bigvee_{r=0}^{n-1} k=n q+r
$$


for all integers $n>1$. The theory of ordered $Z$-groups admits therefore both quantifier elimination and universal axioms in the Presburger language augmented by division with remainder. Thus every definable function is piecewise given by finitely many terms and, consequently, is piecewise linear.

In the residue field sort, we can add new relation symbols for all definable sets and impose suitable postulates. This enables quantifier elimination for the residue field in the augmented language. In this fashion, we have full quantifier elimination in the three-sorted structure $(K, \mathbb{Z}, \mathbb{k})$ with $K=\mathbb{k}((t))$.

Now we can readily pass to the proof of Theorem 3.1 which, of course, reduces easily to the case $m=1$. So let $B$ be an $\mathcal{L}$-definable closed (in the $K$-topology) subset of $D \times R_{y} \subset K_{x}^{n} \times R_{y}$. It suffices to prove that if $a$ lies in the closure of the projection $A:=\pi(B)$, then there is a point $b \in B$ such that $\pi(b)=a$.

Without loss of generality, we may assume that $a=0$. Put

$$
\Lambda:=\left\{\left(v\left(x_{1}\right), \ldots, v\left(x_{n}\right)\right) \in \mathbb{Z}^{n}: x=\left(x_{1}, \ldots, x_{n}\right) \in A\right\}
$$

The set $\Lambda$ contains points all coordinates of which are arbitrarily large, because the point $a=0$ lies in the closure of $A$. Hence and by definable choice, $\Lambda$ contains a set $\Lambda_{0}$ of the form

$$
\Lambda_{0}=\left\{\left(k, \alpha_{2}(k), \ldots, \alpha_{n}(k)\right) \in \mathbb{N}^{n}: k \in \Delta\right\} \subset \Lambda,
$$

where $\Delta \subset \mathbb{N}$ is an unbounded definable subset and

$$
\alpha_{2}, \ldots, \alpha_{n}: \Delta \longrightarrow \mathbb{N}
$$

are increasing unbounded functions given by a term (because a function in one variable given by a term is either increasing or decreasing). We are going to recursively construct a point $b=(0, w) \in B$ with $w \in R$ by performing the following procedure of algorithmic character.

Step 1 Let

$$
\Xi_{1}:=\left\{\left(v\left(x_{1}\right), \ldots, v\left(x_{n}\right), v(y)\right) \in \Lambda_{0} \times \mathbb{N}:(x, y) \in B\right\},
$$

and

$$
\beta_{1}(k):=\sup \left\{l \in \mathbb{N}:\left(k, \alpha_{2}(k), \ldots, \alpha_{n}(k), l\right) \in \Xi_{1}\right\} \in \mathbb{N} \cup\{\infty\}, \quad k \in \Lambda_{0} .
$$

If $\lim \sup _{k \rightarrow \infty} \beta_{1}(k)=\infty$, there is a sequence $\left(x^{(v)}, y^{(v)}\right) \in B, v \in \mathbb{N}$, such that

$$
v\left(x_{1}^{(v)}\right), \ldots, v\left(x_{n}^{(v)}\right), v\left(y^{(v)}\right) \rightarrow \infty
$$

when $v \rightarrow \infty$. Since the set $B$ is a closed subset of $D \times R_{y}$, we get

$$
\left(x^{(v)}, y^{(v)}\right) \rightarrow 0 \in B \quad \text { when } v \rightarrow \infty
$$


and thus $w=0$ is the point we are looking for. Here the process stops. Otherwise

$$
\Lambda_{1} \times\left\{l_{1}\right\} \subset \Xi_{1}
$$

for some infinite definable subset $\Lambda_{1}$ of $\Lambda_{0}$ and $l_{1} \in \mathbb{N}$. The set

$$
\left\{\left(v\left(x_{1}\right), \ldots, v\left(x_{n}\right) ; \overline{a c}(y)\right) \in \Lambda_{1} \times \mathbb{k}:(x, y) \in B, v(y)=l_{1}\right\}
$$

is definable in the language $\mathcal{L}$. By full quantifier elimination, it is given by a quantifierfree formula with variables only from the value group $\Gamma$-sort and the residue field $\mathbb{k}$-sort. Therefore, there is a finite partitioning of $\Lambda_{1}$ into definable subsets over each of which the fibers of the above set are constant, because quantifier-free $\mathcal{L}$-definable subsets of the product $\mathbb{Z}^{n} \times \mathbb{k}$ of the two sorts are finite unions of the Cartesian products of definable subsets in $\mathbb{Z}^{n}$ and in $\mathbb{k}$, respectively. One of those definable subsets, say $\Lambda_{1}^{\prime}$, must be infinite. Consequently, for some $\xi_{1} \in \mathbb{k}$, the set

$$
\Xi_{2}:=\left\{\left(v\left(x_{1}\right), \ldots, v\left(x_{n}\right), v\left(y-\xi_{1} t^{l_{1}}\right)\right) \in \Lambda_{1}^{\prime} \times \mathbb{N}:(x, y) \in B\right\}
$$

contains points of the form $(k, l) \in \mathbb{N}^{n+1}$, where $k \in \Lambda_{1}^{\prime}$ and $l>l_{1}$.

Step 2 Let

$$
\beta_{2}(k):=\sup \left\{l \in \mathbb{N}:\left(k, \alpha_{2}(k), \ldots, \alpha_{n}(k), l\right) \in \Xi_{2}\right\} \in \mathbb{N} \cup\{\infty\}, \quad k \in \Lambda_{1}^{\prime} .
$$

If $\lim \sup _{k \rightarrow \infty} \beta_{2}(k)=\infty$, there is a sequence $\left(x^{(v)}, y^{(v)}\right) \in B, v \in \mathbb{N}$, such that

$$
v\left(x_{1}^{(v)}\right), \ldots, v\left(x_{n}^{(v)}\right), v\left(y^{(v)}-\xi_{1} t^{l_{1}}\right) \rightarrow \infty
$$

when $v \rightarrow \infty$. Since the set $B$ is a closed subset of $D \times R_{y}$, we get

$$
\left(x^{(v)}, y^{(v)}\right) \rightarrow\left(0, \xi_{1} t^{l_{1}}\right) \in B \quad \text { when } v \rightarrow \infty
$$

and thus $w=\xi_{1} t^{l_{1}}$ is the point we are looking for. Here the process stops. Otherwise

$$
\Lambda_{2} \times\left\{l_{2}\right\} \subset \Xi_{2}
$$

for some infinite definable subset $\Lambda_{2}$ of $\Lambda_{1}^{\prime}$ and $l_{2}>l_{1}$. Again, for some $\xi_{2} \in \mathbb{k}$, the set

$$
\Xi_{3}:=\left\{\left(v\left(x_{1}\right), \ldots, v\left(x_{n}\right), v\left(y-\xi_{1} t^{l_{1}}-\xi_{2} t^{l_{2}}\right)\right) \in \Lambda_{2}^{\prime} \times \mathbb{N}:(x, y) \in B\right\}
$$

contains points of the form $(k, l) \in \mathbb{N}^{n+1}$, where $k \in \Lambda_{2}^{\prime}, \Lambda_{2}^{\prime}$ is an infinite definable subset of $\Lambda_{2}$ and $l>l_{2}$.

Step 3 is carried out in the same way as the previous ones; and so on. 
In this fashion, the process either stops after a finite number of steps and then yields the desired point $w \in R$ (actually, $w \in \mathbb{k}[t]$ ) such that $(0, w) \in B$, or it does not stop and then yields a formal power series

$$
w:=\xi_{1} t^{l_{1}}+\xi_{2} t^{l_{2}}+\xi_{3} t^{l_{3}}+\ldots, \quad 0 \leq l_{1}<l_{2}<l_{3}<\ldots
$$

such that for each $v \in \mathbb{N}$ there exists an element $\left(x^{(\nu)}, y^{(v)}\right) \in B$ for which

$$
v\left(y^{(v)}-\xi_{1} t^{l_{1}}-\xi_{2} t^{l_{2}}-\ldots-\xi_{v} t^{l_{v}}\right) \geq l_{v}+1 \geq v, \quad v\left(x_{1}^{(v)}\right), \ldots, v\left(x_{1}^{(v)}\right) \geq v .
$$

Hence $v\left(y^{(v)}-w\right) \geq v$, and thus the sequence $\left(x^{(v)}, y^{(v)}\right)$ tends to the point $b:=(0, w)$ when $v$ tends to $\infty$. Since the set $B$ is a closed subset of $D \times R$, the point $b$ belongs to $B$, which completes the proof.

\section{Definable functions of one variable}

Consider first a complete rank one valued field $L$. For every nonnegative integer $r$, let $L\{x\}_{r}$ be the local ring of all formal power series

$$
\phi(x)=\sum_{k=0}^{\infty} a_{k} x^{k} \in L[[x]]
$$

in one variable $x$ such that $v\left(a_{k}\right)+k r$ tends to $\infty$ when $k \rightarrow \infty ; L\{x\}_{0}$ coincides with the ring of restricted formal power series. Then the local ring

$$
L\{x\}:=\bigcup_{r=0}^{\infty} L\{x\}_{r}
$$

is Henselian, which can be directly deduced by means of the implicit function theorem for restricted power series in several variables (see [7, Chap. III, Sect. 4], [17] and also [19, Chap. I, Sect. 5]).

We keep the assumption that the ground field $K$ is a Henselian rank one valued field of equicharacteristic zero. Let $L$ be the completion of the algebraic closure $\bar{K}$ of $K$. Clearly, the Henselian local ring $L\{x\}$ is closed under division by the coordinate and power substitution. Therefore, it follows from our paper [38, Sect. 2] that Puiseux's theorem holds for $L\{x\}$. We still need an auxiliary lemma.

Lemma 5.1 The field $K$ is a closed subspace of its algebraic closure $\bar{K}$.

Proof This follows directly from that the field $K$ is algebraically maximal (as it is Henselian and finitely ramified; see, e.g., [15, Chap. 4]), but can also be shown as follows. Denote by cl $(E, F)$ the closure of a subset $E$ in $F$, and let $\widehat{K}$ be the completion of $K$. We have

$$
\operatorname{cl}(K, \bar{K})=\operatorname{cl}(K, L) \cap \bar{K}=\widehat{K} \cap \bar{K}
$$


Now, through the transfer principle of Ax-Kochen-Ershov (see, e.g., [9]), $K$ is an elementary substructure of $\widehat{K}$ and, a fortiori, is algebraically closed in $\widehat{K}$. Hence $\operatorname{cl}(K, \bar{K})=\widehat{K} \cap \bar{K}=K$, as asserted.

Now consider an irreducible polynomial

$$
P(x, y)=\sum_{i=0}^{d} p_{i}(x) y^{i} \in K[x, y]
$$

in two variables $x, y$ of $y$-degree $d \geq 1$. Let $Z$ be the Zariski closure of its zero locus in $\bar{K} \times \mathbb{P}^{1}(\bar{K})$. Performing a linear fractional transformation over the ground field $K$ of the variable $y$, we can assume that the fiber $\left\{w_{1}, \ldots, w_{s}\right\}, s \leq d$, of $Z$ over $x=0$ does not contain the point at infinity, i.e., $w_{1}, \ldots, w_{s} \in \bar{K}$. Then $p_{d}(0) \neq 0$ and $p_{d}(x)$ is a unit in $L\{x\}$. Via Hensel's lemma, we get the Hensel decomposition

$$
P(x, y)=p_{d}(x) \cdot \prod_{j=1}^{s} P_{j}(x, y)
$$

of $P(x, y)$ into polynomials

$$
P_{j}(x, y)=\left(y-w_{j}\right)^{d_{j}}+p_{j 1}(x)\left(y-w_{j}\right)^{d_{j-1}}+\cdots+p_{j d_{j}}(x) \in L\{x\}\left[y-w_{j}\right]
$$

which are Weierstrass with respect to $y-w_{j}, j=1, \ldots, s$, respectively. By Puiseux's theorem, there is a neighborhood $U$ of $0 \in \bar{K}$ such that the trace of $Z$ on $U \times \bar{K}$ is a finite union of sets of the form

$$
Z_{\phi_{j}}=\left\{\left(x^{q}, \phi_{j}(x)\right): x \in U\right\} \text { with } \phi_{j} \in L\{x\}, q \in \mathbb{N}, j=1, \ldots, s .
$$

Obviously, for $j=1, \ldots, s$, the fiber of $Z_{\phi_{j}}$ over $x \in U$ tends to the point $\phi_{j}(0)=w_{j}$ when $x \rightarrow 0$.

If $\phi_{j}(0) \in \bar{K} \backslash K$, it follows from Lemma 5.1 that

$$
Z_{\phi_{j}} \cap((U \cap K) \times K)=\emptyset
$$

after perhaps shrinking the neighborhood $U$.

Let us mention that if

$$
\phi_{j}(0) \in K \quad \text { and } \quad \phi_{j} \in L\{x\} \backslash \widehat{K}\{x\}
$$

then

$$
Z_{\phi_{j}} \cap((U \cap K) \times K)=\left\{\left(0, \phi_{j}(0)\right)\right\}
$$


after perhaps shrinking the neighborhood $U$. Indeed, let

$$
\phi_{j}(x)=\sum_{k=0}^{\infty} a_{k} x^{k} \in L[[x]]
$$

and $p$ be the smallest positive integer with $a_{p} \in L \backslash \widehat{K}$. Since $\widehat{K}$ is a closed subspace of $L$, we get

$$
\sum_{k=p}^{\infty} a_{k} x^{k}=x^{p}\left(a_{p}+x \cdot \sum_{k=p+1}^{\infty} a_{k} x^{k-(p+1)}\right) \notin \widehat{K}
$$

for $x$ close enough to 0 , and thus the assertion follows.

Suppose now that an $\mathcal{L}$-definable function $f: A \rightarrow K$ satisfies the equation

$$
P(x, f(x))=0 \text { for } x \in A
$$

and 0 is an accumulation point of the set $A$. It follows immediately from the foregoing discussion that the set $A$ can be partitioned into a finite number of $\mathcal{L}$-definable sets $A_{j}, j=1, \ldots, r$ with $r \leq s$, such that, after perhaps renumbering of the fiber $\left\{w_{1}, \ldots, w_{s}\right\}$ of the set $\{P(x, f(x))=0\}$ over $x=0$, we have

$$
\lim _{x \rightarrow 0} f \mid A_{j}(x)=w_{j} \text { for each } j=1, \ldots, r .
$$

Hence and by Corollary 2.3, we immediately obtain the following

Proposition 5.2 (Existence of the limit) Let $f: A \rightarrow K$ be an $\mathcal{L}$-definable function on a subset $A$ of $K$ and suppose 0 is an accumulation point of $A$. Then there is a finite partition of $A$ into $\mathcal{L}$-definable sets $A_{1}, \ldots, A_{r}$ and points $w_{1} \ldots, w_{r} \in \mathbb{P}^{1}(K)$ such that

$$
\lim _{x \rightarrow 0} f \mid A_{j}(x)=w_{j} \text { for } j=1, \ldots, r \text {. }
$$

Moreover, there is a neighborhood $U$ of 0 such that each definable set

$$
\left\{(v(x), v(f(x))): x \in\left(A_{j} \cap U\right) \backslash\{0\}\right\} \subset \Gamma \times(\Gamma \cup\{\infty\}), j=1, \ldots, r,
$$

is contained in an affine line with rational slope

$$
l=\frac{p_{j}}{q} \cdot k+\beta_{j}, j=1, \ldots, r
$$

with $p_{j}, q \in \mathbb{Z}, q>0, \beta_{j} \in \Gamma$, or in $\Gamma \times\{\infty\}$. 
Remark 5.3 Note that the first conclusion (existence of the limit) could also be established via the lemma on the continuity of roots of a monic polynomial (which can be found in, e.g., [6, Chap. 3, Sect. 4]). Yet another approach for the case of tame theories is provided in [18, Lemma 2.20]. The second conclusion relies on Puiseux's parametrization.

\section{Fiber shrinking for definable sets}

Let $A$ be an $\mathcal{L}$-definable subset of $K^{n}$ with accumulation point

$$
a=\left(a_{1}, \ldots, a_{n}\right) \in K^{n}
$$

and $E$ an $\mathcal{L}$-definable subset of $K$ with accumulation point $a_{1}$. We call an $\mathcal{L}$-definable family of sets

$$
\Phi=\bigcup_{t \in E}\{t\} \times \Phi_{t} \subset A
$$

an $\mathcal{L}$-definable $x_{1}$-fiber shrinking for the set $A$ at $a$ if

$$
\lim _{t \rightarrow a_{1}} \Phi_{t}=\left(a_{2}, \ldots, a_{n}\right)
$$

i.e., for any neighborhood $U$ of $\left(a_{2}, \ldots, a_{n}\right) \in K^{n-1}$, there is a neighborhood $V$ of $a_{1} \in K$ such that $\emptyset \neq \Phi_{t} \subset U$ for every $t \in V \cap E, t \neq a_{1}$. When $n=1, A$ is itself a fiber shrinking for the subset $A$ of $K$ at an accumulation point $a \in K$. This concept is a relaxed version of curve selection. It is used in Sects. 7 and 8 in the proofs of the closedness theorem and a certain version of curve selection.

Proposition 6.1 (Fiber shrinking) Every $\mathcal{L}$-definable subset A of $K^{n}$ with accumulation point $a \in K^{n}$ has, after a permutation of the coordinates, an $\mathcal{L}$-definable $x_{1}$-fiber shrinking at $a$.

Proof We proceed with induction with respect to the dimension of the ambient affine space $n$. The case $n=1$ is trivial. So assuming the assertion to hold for $n$, we shall prove it for $n+1$. We may, of course, assume that $a=0$. Let $x=\left(x_{1}, \ldots, x_{n+1}\right)$ be coordinates in $K_{x}^{n}$.

If 0 is an accumulation point of the intersections

$$
A \cap\left\{x_{i}=0\right\}, \quad i=1, \ldots, n+1,
$$

we are done by the induction hypothesis. Thus we may assume that the intersection

$$
A \cap \bigcup_{i=1}^{n+1}\left\{x_{i}=0\right\}=\emptyset
$$


is empty. Then the definable (in the $\Gamma$-sort) set

$$
P:=\left\{\left(v\left(x_{1}\right), \ldots, v\left(x_{n+1}\right)\right) \in \Gamma^{n+1}: x \in A\right\}
$$

has an accumulation point $(\infty, \ldots, \infty)$.

Since the $\Gamma$-sort admits quantifier elimination in the language of ordered groups augmented by binary relation symbols $\equiv_{n}$ for congruence modulo $n$, every definable subset of $\Gamma^{n+1}$ is a finite union of subsets of semilinear sets contained in $\Gamma^{n+1}$ that are determined by a finite number of congruences

$$
\sum_{j=1}^{n+1} r_{i j} \cdot k_{j} \equiv_{N} \alpha_{i}, \quad i=1, \ldots, s
$$

here $N \in \mathbb{N}, N>1, r_{i j} \in \mathbb{Z}, \alpha_{i} \in \Gamma$ for $i=1, \ldots, s, j=1, \ldots, n+1$.

Consequently, there exists a semilinear subset $P_{0}$ of $\mathbb{R}^{n+1}$ given by finitely many linear equations and inequalities with integer coefficients and with constant terms from $\Gamma$ such that the subset $P_{1}$ of $P_{0} \cap \Gamma^{n+1}$ determined by congruences of the form 6.1 is contained in $P$ and has an accumulation point $(\infty, \ldots, \infty)$. Therefore, there exists an affine semiline

$$
L:=\left\{\left(r_{1} \cdot k+\gamma_{1}, \ldots, r_{n+1} \cdot k+\gamma_{n+1}\right): k \in \Gamma, k \geq 0\right\},
$$

where $r_{1}, \ldots, r_{n+1}$ are positive integers, passing through a point

$$
\left(\gamma_{1}, \ldots, \gamma_{n+1}\right) \in P_{1} \subset \Gamma^{n+1}
$$

and contained in $P_{0}$. It is easy to check that the set

$$
L_{0}:=\left\{\left(\gamma_{1}+r r_{1} N, \ldots, \gamma_{n+1}+r r_{n+1} N\right): r \in \mathbb{N}\right\} \subset P_{1}
$$

is contained in $P_{1}$. Then

$$
\Phi:=\left\{x \in A:\left(v\left(x_{1}\right), \ldots, v\left(x_{n+1}\right)\right) \in L_{0}\right\}
$$

is an $\mathcal{L}$-definable $x_{1}$-fiber shrinking for the set $A$ at 0 . This finishes the proof.

\section{Proof of Theorem 3.1 for the general case}

The proof reduces easily to the case $m=1$. We must show that if $B$ is an $\mathcal{L}$-definable subset of $D \times R$ and a point $a$ lies in the closure of $A:=\pi(B)$, then there is a point $b$ in the closure of $B$ such that $\pi(b)=a$. We may obviously assume that $a=0 \notin A$. By Proposition 6.1, there exists, after a permutation of the coordinates, an $\mathcal{L}$-definable 
$x_{1}$-fiber shrinking $\Phi$ for $A$ at $a$ :

$$
\Phi=\bigcup_{t \in E}\{t\} \times \Phi_{t} \subset A, \quad \lim _{t \rightarrow 0} \Phi_{t}=0
$$

here $E$ is the canonical projection of $A$ onto the $x_{1}$-axis. Put

$$
B^{*}:=\left\{(t, y) \in K \times R: \exists u \in \Phi_{t}(t, u, y) \in B\right\}
$$

it easy to check that if a point $(0, w) \in K^{2}$ lies in the closure of $B^{*}$, then the point $(0, w) \in K^{n+1}$ lies in the closure of $B$. The problem is thus reduced to the case $n=1$ and $a=0 \in K$.

By Corollary 2.7, we can assume that $B$ is a subset $F$ of a cell $C$

$$
F \subset C \subset K_{x} \times R \subset K_{x} \times K_{y}
$$

of the form

$$
\begin{gathered}
F(\xi):=\left\{(x, y) \in K_{x} \times K_{y}:(x, \xi) \in D,\right. \\
v(a(x, \xi)) \triangleleft_{1} v\left((y-c(x, \xi))^{v}\right) \triangleleft_{2} v(b(x, \xi)), \overline{a c}(y-c(x, \xi))=\xi_{1}, \\
\left.v\left(f_{i}(x, \xi)(y-c(x, \xi))^{k_{i}}\right) \equiv_{M} 0, i=1, \ldots, s\right\} .
\end{gathered}
$$

But the set

$$
\left\{(v(x), \xi) \in \Gamma \times \mathbb{k}^{m}: \exists y \in R(x, y) \in F(\xi)\right\}
$$

is an $\mathcal{L}$-definable subset of the product $\Gamma \times \mathbb{k}^{m}$ of the two sorts, which is, by elimination of $K$-quantifiers, a finite union of the Cartesian products of definable subsets in $\Gamma$ and in $\mathbb{k}^{m}$, respectively. It follows that 0 is an accumulation point of the projection $\pi\left(F\left(\xi^{\prime}\right)\right.$ ) of the fiber $F\left(\xi^{\prime}\right)$ for a parameter $\xi^{\prime} \in \mathbb{k}^{m}$. We are thus reduced to the case where $B$ is the fiber $F\left(\xi^{\prime}\right)$ of the set $F$ for a parameter $\xi^{\prime}$. For simplicity, we abbreviate $c\left(x, \xi^{\prime}\right), a\left(x, \xi^{\prime}\right), b\left(x, \xi^{\prime}\right)$ and $f_{i}\left(x, \xi^{\prime}\right)$ to $c(x), a(x), b(x)$ and $f_{i}(x), i=1, \ldots, s$. Denote by $E \subset K$ the domain of these functions; then 0 is an accumulation point of E.

In the statement of Theorem 3.1, we may equivalently replace $R$ with the projective line $\mathbb{P}^{1}(K)$, because the latter is the union of two open and closed charts biregular to $R$. By Proposition 5.2, we can thus assume that the limits, say $c(0), a(0), b(0), f_{i}(0)$ of $c(x), a(x), b(x), f_{i}(x)(i=1, \ldots, s)$ when $x \rightarrow 0$ exist in $\mathbb{P}^{1}(K)$ and, moreover, there is a neighborhood $U$ of 0 such that, each definable set

$$
\left\{\left(v(x), v\left(f_{i}(x)\right)\right): x \in(E \cap U) \backslash\{0\}\right\} \subset \Gamma \times(\Gamma \cup\{\infty\}), i=1, \ldots, s,
$$

is contained in an affine line with rational slope

$$
l=\frac{p_{i}}{q} \cdot k+\beta_{i}, i=1, \ldots, s,
$$


with $p_{i}, q \in \mathbb{Z}, q>0, \beta_{i} \in \Gamma$, or in $\Gamma \times\{\infty\}$.

Performing a linear fractional transformation of the coordinate $y$, we get

$$
c(0), a(0), b(0) \in K \text {. }
$$

The role of the center $c(x)$ is immaterial. We can assume, without loss of generality, that it vanishes, $c(x) \equiv 0$, for if a point $b=(0, w) \in K^{2}$ lies in the closure of the cell with zero center, the point $(0, w+c(0))$ lies in the closure of the cell with center $c(x)$.

When $\triangleleft_{1}$ occurs and $a(0)=0$, the set $F\left(\xi^{\prime}\right)$ is itself an $x$-fiber shrinking at $(0,0)$ and the point $b=(0,0)$ is an accumulation point of $B$ lying over $a=0$, as desired.

So suppose that either only $\triangleleft_{2}$ occurs or $\triangleleft_{1}$ occurs and $a(0) \neq 0$. By elimination of $K$-quantifiers, the set $v(E)$ is a definable subset of $\Gamma$. The value group $\Gamma$ admits quantifier elimination in the language of ordered groups augmented by symbols $\equiv_{n}$ for congruences modulo $n, n \in \mathbb{N}, n>1$ (cf. Sect. 2). Therefore, the set $v(E)$ is of the form

$$
v(E)=\left\{k \in(\alpha, \infty) \cap \Gamma: m_{j} k \equiv_{N} \gamma_{j}, j=1, \ldots, t\right\}
$$

where $\alpha, \gamma_{j} \in \Gamma, m_{j} \in \mathbb{N}$ for $j=1, \ldots, t$.

Now, take an element $(u, w) \in F\left(\xi^{\prime}\right)$ with $u \in(E \cap U) \backslash\{0\}$. By equality 7.2, there is a point $x_{r} \in E, r \in \mathbb{N}$, with

$$
v\left(u_{r}\right)=v(u)+r q M N .
$$

By equality 7.1, we get

$$
v\left(f_{i}\left(u_{r}\right)\right)=v\left(f_{i}(u)\right)+r p_{i} M N, \quad i=1, \ldots, s .
$$

Hence

$$
\begin{aligned}
v\left(f_{i}\left(u_{r}\right) w^{k_{i}}\right) & =v\left(f_{i}\left(u_{r}\right)\right)+k_{i} v(w) \\
& =v\left(f_{i}(u)\right)+r p_{i} M N+k_{i} v(w) \\
& =v\left(f_{i}(u) w^{k_{i}}\right)+r p_{i} M N \equiv_{M} 0 .
\end{aligned}
$$

Of course, after shrinking the neighborhood $U$, we may assume that $v(a(x))=$ $v(a(0))<\infty$ for all $x \in(E \cap U) \backslash\{0\}$. Consequently,

$$
v\left(a\left(u_{r}\right)\right) \triangleleft_{1} v\left(w^{v}\right) \triangleleft_{2} v\left(b\left(u_{r}\right)\right) .
$$

Hence and by 7.3, we get $\left(u_{r}, w\right) \in F\left(\xi^{\prime}\right)$. Since $u_{r}$ tends to $0 \in K$ when $r \rightarrow \infty$, the point $(0, w)$ is an accumulation point of $F\left(\xi^{\prime}\right)$ lying over $0 \in K$, which completes the proof of the closedness theorem. 


\section{Curve selection}

We now pass to curve selection over non-locally compact ground fields under study. While the real version of curve selection goes back to the papers $[8,45]$ (see also [5, $35,36]$ ), the $p$-adic one was achieved in the papers $[14,44]$. Before proving a general version for $\mathcal{L}$-definable sets, we give a version for valuative semialgebraic sets. Our approach relies on resolution of singularities, which was already suggested by Denefvan den Dries [14] in the remark after Theorem 3.34.

By a valuative semialgebraic subset of $K^{n}$, we mean a (finite) Boolean combination of elementary valuative semialgebraic subsets, i.e., sets of the form

$$
\left\{x \in K^{n}: v(f(x)) \leq v(g(x))\right\},
$$

where $f$ and $g$ are regular functions on $K^{n}$. We call a map $\varphi$ semialgebraic if its graph is a valuative semialgebraic set.

Proposition 8.1 Let $A$ be a valuative semialgebraic subset of $K^{n}$. If a point a $\in K^{n}$ lies in the closure (in the $K$-topology) of $A \backslash\{a\}$, then there is a semialgebraic map $\varphi: R \longrightarrow K^{n}$ given by restricted power series such that

$$
\varphi(0)=a \text { and } \varphi(R \backslash\{0\}) \subset A \backslash\{a\} .
$$

Proof It is easy to check that every valuative semialgebraic set is a finite union of basic valuative semialgebraic sets, i.e., sets of the form

$$
\left\{x \in K^{n}: v\left(f_{1}(x)\right) \triangleleft_{1} v\left(g_{1}(x)\right), \ldots, v\left(f_{r}(x)\right) \triangleleft_{r} v\left(g_{r}(x)\right)\right\},
$$

where $f_{1}, \ldots, f_{r}, g_{1}, \ldots, g_{r}$ are regular functions and $\triangleleft_{1}, \ldots, \triangleleft_{r}$ stand for $\leq$ or $<$. We may assume, of course, that $A$ is a set of this form and $a=0$. Take a finite composite

$$
\sigma: Y \longrightarrow K \mathbb{A}^{n}
$$

of blowups along smooth centers such that the pullbacks of the coordinates $x_{1}, \ldots, x_{n}$ and the pullbacks

$$
f_{1}^{\sigma}:=f_{1} \circ \sigma, \ldots, f_{r}^{\sigma}:=f_{r} \circ \sigma \text { and } g_{1}^{\sigma}:=g_{1} \circ \sigma, \ldots, g_{r}^{\sigma}:=g_{r} \circ \sigma
$$

are normal crossing divisors ordered with respect to divisibility relation, unless they vanish. Since the restriction $\sigma: Y(K) \longrightarrow K^{n}$ is definably closed (Corollary 3.5), there is a point $b \in Y(K) \cap \sigma^{-1}(a)$ which lies in the closure of the set

$$
B:=Y(K) \cap \sigma^{-1}(A \backslash\{a\}) .
$$

Further, we get

$$
Y(K) \cap \sigma^{-1}(A)=\left\{v\left(f_{1}^{\sigma}(y)\right) \triangleleft_{1} v\left(g_{1}^{\sigma}(y)\right)\right\} \cap \ldots \cap\left\{v\left(f_{r}^{\sigma}(y)\right) \triangleleft_{r} v\left(g_{r}^{\sigma}(y)\right)\right\},
$$


and thus $\sigma^{-1}(A)$ is in suitable local coordinates $y=\left(y_{1}, \ldots, y_{n}\right)$ near $b=0$ a finite intersection of sets of the form

$$
\left\{v\left(y^{\alpha}\right) \leq v(u(y))\right\},\left\{v(u(y)) \leq v\left(y^{\alpha}\right)\right\},\left\{v\left(y^{\beta}\right)<\infty\right\} \text { or }\left\{\infty=v\left(y^{\gamma}\right)\right\}
$$

where $\alpha, \beta, \gamma \in \mathbb{N}^{n}$ and $u(y)$ is a regular, nowhere vanishing function.

The first case cannot occur because $b=0$ lies in the closure of $B$; the second case holds in a neighborhood of $b$; the third and fourth cases are equivalent to $y^{\beta} \neq 0$ and $y^{\gamma}=0$, respectively. Consequently, since the pullbacks of the coordinates $x_{1}, \ldots, x_{n}$ are monomial divisors too, $B$ contains the set $(R \backslash\{0\}) \cdot c$ when $c \in B$ is a point sufficiently close to $b=0$. Then the map

$$
\varphi: R \longrightarrow K^{n}, \quad \varphi(z)=\sigma(z \cdot c)
$$

has the desired properties.

We now pass to the general version of curve selection for $\mathcal{L}$-definable sets.

Proposition 8.2 Let $A$ be an $\mathcal{L}$-definable set subset of $K^{n}$. If a point a $\in K^{n}$ lies in the closure (in the $K$-topology) of $A \backslash\{a\}$, then there exist a semialgebraic map $\varphi: R \longrightarrow K^{n}$ given by restricted power series and an $\mathcal{L}$-definable subset $E$ of $R$ with accumulation point 0 such that

$$
\varphi(0)=a \text { and } \varphi(E \backslash\{0\}) \subset A \backslash\{a\} .
$$

Proof We proceed with induction with respect to the dimension of the ambient space $n$. The case $n=1$ being evident, suppose $n>1$. By elimination of $K$-quantifiers, similarly as in Sect. 2, the set $A \backslash\{a\}$ is a finite union of sets defined by conditions of the form

$$
\left(v\left(f_{1}(x)\right), \ldots, v\left(f_{r}(x)\right)\right) \in P, \quad\left(\overline{a c} g_{1}(x), \ldots, \overline{a c} g_{s}(x)\right) \in Q,
$$

where $f_{i}, g_{j} \in K[x]$ are polynomials, and $P$ and $Q$ are definable subsets of $\Gamma^{r}$ and $\mathbb{K}^{s}$, respectively ( and, of course, that $a=0$.

Again, take a finite composite

$$
\sigma: Y \longrightarrow K \mathbb{A}^{n}
$$

of blowups along smooth centers such that the pullbacks

$$
f_{1}^{\sigma}, \ldots, f_{r}^{\sigma} \text { and } g_{1}^{\sigma}, \ldots, g_{r}^{\sigma}
$$

are normal crossing divisors unless they vanish. Since the restriction $\sigma: Y(K) \longrightarrow$ $K^{n}$ is definably closed (Corollary 3.5), there is a point $b \in Y(K) \cap \sigma^{-1}(a)$ which lies in the closure of the set

$$
B:=Y(K) \cap \sigma^{-1}(A \backslash\{a\}) .
$$


Take local coordinates $y_{1} \ldots, y_{n}$ near $b$ in which $b=0$ and every pullback above is a normal crossing. We shall first select a semialgebraic map $\psi: R \longrightarrow Y(K)$ given by restricted power series and an $\mathcal{L}$-definable subset $E$ of $R$ with accumulation point 0 such that

$$
\psi(0)=b \text { and } \psi(E \backslash\{0\}) \subset B .
$$

Since the valuation map and the angular component map composed with a continuous function are locally constant near any point at which this function does not vanish, the conditions which describe the set $B$ near $b$ are of the form

$$
\left(v\left(y_{1}\right), \ldots, v\left(y_{n}\right)\right) \in \widetilde{P}, \quad\left(\overline{a c} y_{1}, \ldots, \overline{a c} y_{n}\right) \in \widetilde{Q},
$$

where $\widetilde{P}$ and $\widetilde{Q}$ are definable subsets of $\Gamma^{n}$ and $\mathbb{k}^{n}$, respectively.

The set $B_{0}$ determined by the conditions

$$
\begin{aligned}
& \left(v\left(y_{1}\right), \ldots, v\left(y_{n}\right)\right) \in \widetilde{P}, \\
& \left(\overline{a c} y_{1}, \ldots, \overline{a c} y_{n}\right) \in \widetilde{Q} \cap \bigcup_{i=1}^{n}\left\{\xi_{i}=0\right\},
\end{aligned}
$$

is contained near $b$ in the union of hyperplanes $\left\{y_{i}=0\right\}, i=1, \ldots, n$. If $b$ is an accumulation point of the set $B_{0}$, then the desired map $\psi$ exists by the induction hypothesis. Otherwise $b$ is an accumulation point of the set $B_{1}:=B \backslash B_{0}$.

Analysis from the proof of Proposition 6.1 (fiber shrinking) shows that the congruences describing the definable subset $\widetilde{P}$ of $\Gamma^{n}$ are not an essential obstacle to finding the desired map $\psi$, but affect only the definable subset $E$ of $R$. Neither are the conditions

$$
\widetilde{Q} \backslash \bigcup_{i=1}^{n}\left\{\xi_{i}=0\right\}
$$

imposed on the angular components of the coordinates $y_{1}, \ldots, y_{n}$, because then none of them vanishes. Therefore, in order to select the map $\psi$, we must first of all analyze the linear conditions (equalities and inequalities) describing the set $\widetilde{P}$.

The set $\widetilde{P}$ has an accumulation point $(\infty, \ldots, \infty)$ as $b=0$ is an accumulation point of $B$. We see, similarly as in the proof of Proposition 6.1 (fiber shrinking), that $\widetilde{P}$ contains a definable subset of a semiline

$$
L:=\left\{\left(r_{1} \cdot k+\gamma_{1}, \ldots, r_{n} \cdot k+\gamma_{n}\right): k \in \Gamma, k \geq 0\right\},
$$

where $r_{1}, \ldots, r_{n}$ are positive integers, passing through a point

$$
\gamma_{1}, \ldots, \gamma_{n} \in \widetilde{P} \subset \Gamma^{n}
$$

clearly, $(\infty, \ldots, \infty)$ is an accumulation point of that definable subset of $L$. 
Now, take some elements

$$
\left(\xi_{1}, \ldots, \xi_{n}\right) \in \widetilde{Q} \backslash \bigcup_{i=1}^{n}\left\{\xi_{i}=0\right\}
$$

and next some elements $w_{1}, \ldots, w_{n} \in K$ for which

$$
v\left(w_{1}\right)=\gamma_{1}, \ldots, v\left(w_{n}\right)=\gamma_{n} \text { and } \overline{a c} w_{1}=\xi_{1}, \ldots, \overline{a c} w_{n}=\xi_{n} .
$$

There exists an $\mathcal{L}$-definable subset $E$ of $R$ which is determined by some congruences imposed on $v(t)$ (as in the proof of Proposition 6.1) and the conditions $\overline{a c} t=1 \mathrm{such}$ that the subset

$$
F:=\left\{\left(w_{1} \cdot t^{r_{1}}, \ldots, w_{n} \cdot t^{r_{n}}\right): t \in E\right\}
$$

of the arc

$$
\psi: R \rightarrow Y, \quad \psi(t)=\left(w_{1} \cdot t^{r_{1}}, \ldots, w_{n} \cdot t^{r_{n}}\right)
$$

is contained in $B_{1}$. Then $\varphi:=\sigma \circ \psi$ is the map we are looking for. This completes the proof.

\section{Lojasiewicz inequality}

In this section, we provide certain general versions of the Łojasiewicz inequality. For the classical version over the real ground field, we refer the reader to [5, Thm. 2.6.6].

Proposition 9.1 Let $f, g: A \rightarrow K$ be two continuous $\mathcal{L}$-definable functions on a closed (in the $K$-topology) $\mathcal{L}$-definable subset $A$ of $R^{m}$. If

$$
\{x \in A: g(x)=0\} \subset\{x \in A: f(x)=0\},
$$

then there exist a positive integer $s$ and a continuous $\mathcal{L}$-definable function $h$ on $A$ such that $f^{s}(x)=h(x) \cdot g(x)$ for all $x \in A$.

Proof It is easy to check that the set

$$
A_{\gamma}:=\{x \in A: v(f(x))=\gamma\}
$$

is a closed $\mathcal{L}$-definable subset of $A$ for every $\gamma \in \Gamma$. Hence and by Corollary 3.3 to the closedness theorem, the set $g\left(A_{\gamma}\right)$ is a closed $\mathcal{L}$-definable subset of $K \backslash\{0\}, \gamma \in \Gamma$. The set $v\left(g\left(A_{\gamma}\right)\right)$ is thus bounded from above, i.e.,

$$
v\left(g\left(A_{\gamma}\right)\right)<\alpha(\gamma)
$$


for some $\alpha(\gamma) \in \Gamma$. By elimination of $K$-quantifiers, the set

$$
\Lambda:=\left\{(v(f(x)), v(g(x))) \in \Gamma^{2}: x \in A\right\} \subset\left\{(\gamma, \delta) \in \Gamma^{2}: \delta<\alpha(\gamma)\right\}
$$

is a definable subset of $\Gamma^{2}$, and thus it is described by a finite number of linear inequalities and congruences. Hence

$$
\Lambda \cap\left\{(\gamma, \delta) \in \Gamma^{2}: \gamma>\gamma_{0}\right\} \subset\left\{(\gamma, \delta) \in \Gamma^{2}: \delta<s \cdot \gamma\right\}
$$

for a positive integer $s$ and some $\gamma_{0} \in \Gamma$. We thus get

$$
v(g(x))<s \cdot v(f(x)) \text { if } x \in A, v(f(x))>\gamma_{0},
$$

whence

$$
v(g(x))<v\left(f^{s}(x)\right) \text { if } x \in A, v(f(x))>\gamma_{0} .
$$

Consequently, the quotient $f^{s} / g$ extends by zero through the zero set of the denominator to a (unique) continuous $\mathcal{L}$-definable function on $A$. This finishes the proof.

The above theorem can be generalized as follows.

Proposition 9.2 Let $U$ and $F$ be two $\mathcal{L}$-definable subsets of $K^{m}$, suppose $U$ is open and $F$ closed in the $K$-topology and consider two continuous $\mathcal{L}$-definable functions $f, g: A \rightarrow K$ on the locally closed subset $A:=U \cap F$ of $K^{m}$. If

$$
\{x \in A: g(x)=0\} \subset\{x \in A: f(x)=0\},
$$

then there exist a positive integer $s$ and a continuous $\mathcal{L}$-definable function $h$ on $A$ such that $f^{s}(x)=h(x) \cdot g(x)$ for all $x \in A$.

Proof We shall adapt the foregoing arguments. Since the set $U$ is open, its complement $V:=K^{m} \backslash U$ is closed in $K^{m}$ and $A$ is the following union of open and closed subsets of $K^{m}$ and of $\mathbb{P}^{m}(K)$ :

$$
\begin{array}{r}
X_{\beta}:=\left\{x \in K^{m}: v\left(x_{1}\right), \ldots, v\left(x_{m}\right) \geq-\beta,\right. \\
v(x-y) \leq \beta \text { for all } y \in V\},
\end{array}
$$

where $\beta \in \Gamma, \beta \geq 0$. As before, we see that the sets

$$
A_{\beta, \gamma}:=\left\{x \in X_{\beta}: v(f(x))=\gamma\right\} \text { with } \beta, \gamma \in \Gamma
$$

are closed $\mathcal{L}$-definable subsets of $\mathbb{P}^{m}(K)$, and next that the sets $g\left(A_{\beta, \gamma}\right)$ are closed $\mathcal{L}$-definable subsets of $K \backslash\{0\}$ for all $\beta, \gamma \in \Gamma$. Likewise, we get

$$
\begin{aligned}
& \Lambda:=\left\{(\beta, v(f(x)), v(g(x))) \in \Gamma^{3}: x \in X_{\beta}\right\} \subset \\
& \quad \subset\left\{(\beta, \gamma, \delta) \in \Gamma^{3}: \delta<\alpha(\beta, \gamma)\right\}
\end{aligned}
$$


for some $\alpha(\beta, \gamma) \in \Gamma$. Consequently, since $\Lambda$ is a definable subset of $\Gamma^{3}$, there exist a positive integer $s$ and elements $\gamma_{0}(\beta) \in \Gamma$ such that

$$
\Lambda \cap\left\{(\beta, \gamma, \delta) \in \Gamma^{3}: \gamma>\gamma_{0}(\beta)\right\} \subset\left\{(\beta, \gamma, \delta) \in \Gamma^{3}: \delta<s \cdot \gamma\right\}
$$

Since $A$ is the union of the sets $X_{\beta}$, it is not difficult to check that the quotient $f^{s} / g$ extends by zero through the zero set of the denominator to a (unique) continuous $\mathcal{L}$-definable function on $A$, which is the desired result.

\section{Extending continuous hereditarily rational functions}

We first recall an elementary lemma from [27, Lemma 15].

Lemma 10.1 If the ground field $K$ is not algebraically closed, then there are polynomials $G_{r}\left(x_{1}, \ldots, x_{r}\right)$ in any number of variables whose only zero on $K^{r}$ is $(0, \ldots, 0)$. In particular,

$$
G_{2}\left(x_{1}, x_{2}\right)=x_{1}^{d}+a_{1} x_{1}^{d-1} x_{2}+\cdots+a_{d} x_{2}^{d},
$$

where

$$
t^{d}+a_{1} t^{d-1}+\cdots+a_{d} \in K[t]
$$

is a polynomial with no roots in $K$.

We keep further the assumption that $K$ is a Henselian rank one valued field of equicharacteristic zero and, additionally, that it is not algebraically closed. We have at our disposal the descent property (Corollary 3.6) and the Łojasiewicz inequality (Proposition 9.2). Therefore, by adapting mutatis mutandis its proof, we are able to carry over Proposition 11 from [27] on extending continuous hereditarily rational functions (being its main extension result) to the case of such non-archimedean fields. We first recall the definition. Given a $K$-variety $Z$, we say that a continuous function $f: Z(K) \rightarrow K$ is hereditarily rational if every irreducible subvariety $Y \subset Z$ has a Zariski dense open subvariety $Y^{0} \subset Z$ such that $\left.f\right|_{Y^{0}(K)}$ is regular.

Theorem 10.2 Let $X$ be a smooth $K$-variety and $W \subset Z \subset X$ closed subvarieties. Let $f$ be a continuous hereditarily rational function on $Z(K)$ that is regular at all $K$ points of $Z(K) \backslash W(K)$. Then $f$ extends to a continuous hereditarily rational function $F$ on $X(K)$ that is regular at all $K$-points of $X(K) \backslash W(K)$.

Remark 10.3 The corresponding theorem for differentiable hereditarily rational functions remains an open problem as yet (cf. Remark 13.9 and the discussion afterward).

Sketch of the Proof We shall keep the notation from the paper [27]. The main modification of the proof in comparison with that paper is the definition of the functions 
$G$ and $F_{2 n}$ which improve the rational function $P / Q$. Now we need the following corrections:

$$
G:=\frac{P}{Q} \cdot \frac{Q^{d}}{G_{2}(Q, H)}
$$

and

$$
F_{d n}:=G \cdot \frac{Q^{d n}}{G_{2}\left(Q^{n}, H\right)}=\frac{P}{Q} \cdot \frac{Q^{d}}{G_{2}(Q, H)} \cdot \frac{Q^{d n}}{G_{2}\left(Q^{n}, H\right)},
$$

where the positive integer $d$ and the polynomial $G_{2}$ are taken from Lemma 10.1. It is clear that the restriction of $F_{d n}$ to $Z \backslash W$ equals $f_{2}$, and thus Theorem 10.2 will be proven once we show that the rational function $F_{d n}$ restricts to a continuous function $\Phi_{d n}$ on $X(K)$ for $n \gg 1$.

We work on the variety $\pi: X_{1}(K) \longrightarrow X(K)$ obtained by blowing up the ideal $\left(P Q^{d-1}, G_{2}(Q, H)\right)$; let $E:=\pi^{-1}(W)$ be its exceptional divisor. Equivalently, $X_{1}(K)$ is the Zariski closure of the graph of $G$ in $X(K) \times \mathbb{P}^{1}(K)$. Two open charts are considered:

- a Zariski open neighborhood $U^{*}$ of the closure (in the $K$-topology) $Z^{*}$ of $\pi^{-1}(Z(K) \backslash W(K))$;

- an open (in the $K$-topology) set $V^{*}:=X_{1}(K) \backslash Z^{*}$, which is an $\mathcal{L}$-definable subset of $X_{1}(K)$.

Via the descent property (Corollary 3.6), it suffices to show that the rational function $F_{d n} \circ \pi$ (with $n \gg 1$ ) extends to a continuous function on $X_{1}(K)$ that vanishes on $E(K)$.

The subtlest analysis is on the latter chart, on which $F_{d n} \circ \pi$ can be written in the form

$$
F_{d n} \circ \pi=(P \circ \pi) \cdot\left(\frac{Q^{d n-1}}{H^{d}} \circ \pi\right) \cdot\left(\frac{Q^{d}}{G_{2}(Q, H)} \circ \pi\right) \cdot\left(\frac{H^{d}}{G_{2}\left(Q^{n}, H\right)} \circ \pi\right) .
$$

Note that on $V^{*}$ the function $H \circ \pi$ vanishes only along $E(K)$ and $Q \circ \pi$ vanishes along $E(K)$ too. Therefore, we can apply Proposition 9.2 (Łojasiewicz inequality) to the numerator and denominator of the first factor, which are regular functions on the chart $V^{*}$, to immediately deduce that the first factor extends to a continuous rational function on $V^{*}$ that vanishes along $E(K) \cap V^{*}$ for $n \gg 1$.

What still remains to prove (cf. [27]) is that the factors

$$
\frac{Q^{d n}}{G_{2}\left(Q^{n}, H\right)}, \quad \frac{Q^{d}}{G_{2}(Q, H)} \text { and } \frac{H^{d}}{G_{2}\left(Q^{n}, H\right)}
$$

are regular functions off $W(K)$ whose valuations are bounded from below. But this follows immediately from an auxiliary lemma: 
Lemma 10.4 Let $g$ be the polynomial from the proof of Lemma 10.1. Then the set of values

$$
v\left(\frac{t^{d}}{g(t)}\right) \in \Gamma, \quad t \in K
$$

is bounded from below.

In order to prove this lemma, observe that

$$
v\left(\frac{t^{d}}{g(t)}\right)=v\left(1+\frac{a_{1}}{t}+\cdots+\frac{a_{d}}{t^{d}}\right)
$$

for $t \in K, t \neq 0$. Hence the values under study are zero if $i v(t)<v\left(a_{i}\right)$ for all $i=1, \ldots, d$. Therefore, we are reduced to analyzing the case where

$$
v(t) \geq k:=\min \left\{\frac{v\left(a_{i}\right)}{i}: i=1, \ldots, d\right\} .
$$

Denote by $\Gamma$ the value group of $v$. Thus we must show that the set of values $v(g(t)) \in \Gamma$ when $v(t) \geq k$ is bounded from above.

Take elements $a, b \in R, a, b \neq 0$, such that $a a_{i} \in R$ for all $i=1, \ldots, d$, and $b t \in R$ whenever $v(t) \geq k$. Then

$$
\begin{aligned}
& h(a b t):=(a b)^{d} g(t) \\
& \quad=(a b t)^{d}+a b a_{1}(a b t)^{d-1}+(a b)^{2} a_{2}(a b t)^{d-2}+\cdots+(a b)^{d} a_{d}
\end{aligned}
$$

is a monic polynomial with coefficients from $R$ which has no roots in $K$. Clearly, it is sufficient to show that the set of values $v(h(t)) \in \Gamma$ when $t \in R$ is bounded from above.

Consider a splitting field $\widetilde{K}=K\left(u_{1}, \ldots, u_{d}\right)$ of the polynomial $h$, where $u_{1}, \ldots, u_{d}$ are the roots of $h$. Let $\widetilde{v}$ be a (unique) extension to $\widetilde{K}$ of the valuation $v, \widetilde{R}$ be its valuation ring and $\widetilde{\Gamma} \supset \Gamma$ its value group (see, e.g., [46, Chap. VI, Sect. 11] for valuations of algebraic field extensions). Then

$$
u_{1}, \ldots, u_{d} \in \widetilde{R} \backslash R \text { and } h(t)=\prod_{i=1}^{d}\left(t-u_{i}\right) .
$$

Since $R$ is a closed subring of $\widetilde{R}$ by Lemma 5.1, there exists an $l \in \widetilde{\Gamma}$ such that $\widetilde{v}\left(t-u_{i}\right) \leq l$ for all $i=1, \ldots, d$ and $t \in R$. Hence $v(h(t)) \leq d l$ for all $t \in R$, and thus the lemma follows.

In this fashion, we have demonstrated how to adapt the proof of Proposition 11 from [27] to the case of Henselian rank one valued fields of equicharacteristic zero. Note that the proofs of all remaining results from that paper work over general topological fields with a density property introduced in [27, Sect. 3] and recalled below. 
A topological field $K$ satisfies the density property if one of the following equivalent conditions holds:

(1) If $X$ is a smooth, irreducible $K$-variety and $\emptyset \neq U \subset X$ is a Zariski open subset, then $U(K)$ is dense in $X(K)$ in the $K$-topology.

(2) If $C$ is a smooth, irreducible $K$-curve and $\emptyset \neq C^{0} \subset C$ is a Zariski open subset, then $C^{0}(K)$ is dense in $C(K)$ in the $K$-topology.

(3) If $C$ is a smooth, irreducible $K$-curve, then $C(K)$ has no isolated points.

The examples of such fields are, in particular, all Henselian rank one valued fields.

\section{Regulous functions and sets}

In these last three sections, we shall carry the theory of regulous functions over the real ground field $\mathbb{R}$, developed by Fichou-Huisman-Mangolte-Monnier [16], over to non-archimedean algebraic geometry over Henselian rank one valued fields $K$ of equicharacteristic zero. We assume that the ground field $K$ is not algebraically closed. (Otherwise, the notion of a regulous function on a normal variety coincides with that of a regular function and, in general, the study of continuous rational functions leads to the concept of seminormality and seminormalization; cf. [1,2] or [26, Sect. 10.2] for a recent treatment.) Every such field enjoys the density property. The $K$-points $X(K)$ of any algebraic $K$-variety $X$ inherit from $K$ a topology, called the $K$-topology.

In this section, we deal with the ground fields $K$ with the density property. Observe first that if $f$ is a rational function on an affine $K$-variety $X$ which is regular on a Zariski open subset $U$, then there exist two regular functions $p, q$ on $X$ such that

$$
f=\frac{p}{q} \text { and } q(x) \neq 0 \text { for all } x \in U(K) .
$$

When $X \subset K \mathbb{A}^{n}$, then $p, q$ can be polynomial functions. For every rational function $f$ on $X$, there is a largest Zariski open subset of $X$ on which $f$ is regular, called the regular locus of $f$ and denoted by $\operatorname{dom}(f)$. Further, assume that $Z$ is a closed subvariety of a $K$-variety $X$. Then every rational function $f$ on $Z$ that is regular on $Z(K)$ extends to a rational function $F$ on $X$ that is regular on $X(K)$. Both the results can be deduced via Lemma 10.1 (cf. [27], the proof of Lemma 15 on extending regular functions).

Suppose now that $X$ is a smooth affine $K$-variety or, at least, an affine $K$-variety that is smooth at all $K$-points $X(K)$. We say that a function $f$ on $X(K)$ is $k$-regulous, $k \in \mathbb{N} \cup\{\infty\}$, if it is of class $\mathcal{C}^{k}$ and there is a Zariski dense open subset $U$ of $X$ such that the restriction of $f$ to $U(K)$ is a regular function. A function $f$ on $X(K)$ is called regulous if it is 0-regulous. Denote by $\mathcal{R}^{k}(X(K))$ the ring of $k$-regulous functions on $X(K)$. The ring $\mathcal{R}^{\infty}(X(K))$ of $\infty$-regulous functions on $X(K)$ coincides with the ring $\mathcal{O}(X(K))$ of regular functions on $X(K)$. This follows easily from the faithful flatness of the formal power series ring $K\left[\left[x_{1}, \ldots, x_{n}\right]\right]$ over the local ring of regular function germs at $0 \in K^{n}$. Therefore, we shall restrict ourselves to the case $k \in \mathbb{N}$. 
When $K$ is a Henselian rank one valued field of equicharacteristic zero, transformation to a normal crossing by blowing up along smooth centers and the descent property (Corollary 3.6) enable the following characterization:

Given a smooth algebraic $K$-variety $X$, a function

$$
f: X(K) \rightarrow K
$$

is regulous iff there exists a finite composite $\sigma: \widetilde{X} \rightarrow X$ of blowups with smooth centers such that the pullback $f^{\sigma}:=f \circ \sigma$ is a regular function on $\widetilde{X}(K)$.

We say that a subset $V$ of $K^{n}$ is $k$-regulous closed if it is the zero set of a family $E \subset \mathcal{R}^{k}\left(\mathbb{K}^{n}\right)$ of $k$-regulous functions:

$$
V=\mathcal{Z}(E):=\left\{x \in K^{n}: f(x)=0 \text { for all } f \in E\right\}
$$

A subset $U$ of $K^{n}$ is called $k$-regulous open if its complement $\mathbb{K}^{n} \backslash U$ is $k$-regulous closed. The family of $k$-regulous open subsets of $K^{n}$ is a topology on $K^{n}$, called the $k$-regulous topology on $K^{n}$.

If $f \neq 0$ is a $k$-regulous function on $K^{n}$ with regular locus

$$
U=\operatorname{dom}(f) \subset K^{n},
$$

then

$$
f=\frac{p}{q} \text { where } p, q \in K\left[x_{1}, \ldots, x_{n}\right], \mathcal{Z}(q)=K^{n} \backslash U(K)
$$

and $p, q$ are coprime polynomials. Clearly, $\mathcal{Z}(q) \subset \mathcal{Z}(p)$ and it follows, by passage to the algebraic closure of $K$, that the zero set $\mathcal{Z}(q)$ is of codimension $\geq 2$ in $K^{n}$. Thus the complement $K^{n} \backslash \operatorname{dom}(f)$ is of codimension $\geq 2$ in $K^{n}$. Consequently, every $k$-regulous function on $K$ is regular and every $k$-regulous function on $K^{2}$ is regular at all but finitely many points.

We now recall some results about algebraic varieties over arbitrary fields $F$. Let $V$ be an affine $F$-variety. We are interested in the set $V(F)$ of its $F$-points. Therefore, from now on, we shall (and may) assume that $V(F)$ is Zariski dense in $V$. Then the regular locus Reg $(V)$ of $V$ is a non-empty, Zariski open subset of $V$ and, moreover, its trace on the set $V(F)$ is non-empty; cf. [32], Chap. VI, Corollary 1.17 to the Jacobian criterion for regular local rings and the remark preceding it. If the ground field $F$ is not algebraically closed, then the trace $\operatorname{Reg}(V) \cap V(F)$ is smooth and affine, because it follows immediately from Lemma 10.1 that every algebraic subset of $F^{n}$ is the zero set of one polynomial. Summing up, we see that, for every affine $F$-subvariety $V$ of $F \mathbb{A}^{n}$, the set $V(F) \subset F^{n}$ of its $F$-points is a finite (disjoint) union of smooth, affine, Zariski locally closed subsets of $F^{n}$ of pure dimension.

We call a subset $E$ of $K^{n}$ constructible if it is a (finite) Boolean combination of Zariski closed subsets of $K^{n}$. Every such set $E$ is, of course, a finite union of Zariski locally closed subsets. Further, in view of the foregoing discussion, $E$ is a finite union of smooth, affine, Zariski locally closed subsets of pure dimension with irreducible 
Zariski closure. Thus it follows immediately from the density property that every closed (in the $K$-topology) constructible subset $E$ of $K^{n}$ is a finite union of a unique irredundant family $\Sigma(E)$ of constructible subsets each of which is the regular locus $\operatorname{Reg}(V) \cap K^{n}$ of an irreducible affine $K$-subvariety $V$ of $K \mathbb{A}^{n} ;$ obviously, $\operatorname{Reg}(V) \cap K^{n}$ is a smooth, Zariski locally closed subset of pure dimension $\operatorname{dim} V$.

Below we introduce the constructible topology on $K^{n}$. We shall see in the next section that the $k$-regulous topology coincides with the constructible topology for all $k \in \mathbb{N}$.

Proposition 11.1 If $K$ is a topological field with the density property, then the family of all closed (in the $K$-topology) constructible subsets of $K^{n}$ is the family of closed sets for a topology, called the constructible topology on $K^{n}$. Furthermore, this topology is noetherian, i.e., every descending sequence of closed constructible subsets of $K^{n}$ stabilizes.

Proof Clearly, it suffices to prove only the last assertion. We shall follow the reasoning from our paper [37] which showed that the quasi-analytic topology is noetherian. For any closed (in the $K$-topology) constructible subset $E$ of $K^{n}$, let $\mu_{i}(E)$ be the number of elements from the family $\Sigma(E)$, constructed above, of dimension $i, i=0,1, \ldots, n$, and put

$$
\mu(E)=\left(\mu_{n}(E), \mu_{n-1}(E), \ldots, \mu_{0}(E)\right) \in \mathbb{N}^{n+1}
$$

Consider now a descending sequence of closed constructible subsets

$$
K^{n} \supset E_{1} \supset E_{2} \supset E_{3} \supset \ldots
$$

It is easy to check that for any two closed (in the $K$-topology) constructible subsets $D \subset E$, we have $\mu(D) \leq \mu(E)$ and, furthermore, $D=E$ iff $\mu(D)=\mu(E)$. Hence we get the decreasing (in the lexicographic order) sequence of multi-indices

$$
\mu\left(E_{1}\right) \geq \mu\left(E_{2}\right) \geq \mu\left(E_{3}\right) \geq \ldots,
$$

which must stabilize for some $N \in \mathbb{N}$ :

$$
\mu\left(E_{N}\right)=\mu\left(E_{N+1}\right)=\mu\left(E_{N+2}\right)=\ldots
$$

Then

$$
E_{N}=E_{N+1}=E_{N+2}=\ldots,
$$

as desired.

Corollary 11.2 Suppose $K$ is a field with the density property. Then there is a oneto-one correspondence between the irreducible closed constructible subsets $E$ of $K^{n}$ and the irreducible Zariski closed subsets $V$ of $K^{n}$ :

$$
\alpha: E \longmapsto \bar{E}^{Z} \text { and } \beta: V \longmapsto \overline{\operatorname{Reg}(V)}^{c} \text {, }
$$


where $\bar{E}^{Z}$ stands for the Zariski closure of $E$ and $\bar{A}^{c}$ for the closure of $A$ in the constructible topology.

Proof We have $\alpha \circ \beta=$ Id, because $\overline{\operatorname{Reg}(V)}^{Z}=V$ for every irreducible Zariski closed subset $V$ of $K^{n}$. In view of the foregoing discussion, the assignment $\beta$ is surjective. Therefore, every irreducible closed constructible subset $E$ of $K^{n}$ is of the form $E=\beta(V)=\overline{\operatorname{Reg}(V)}^{c}$ for an irreducible Zariski closed subset $V$ of $K^{n}$. Hence

$$
(\beta \circ \alpha)(E)=(\beta \circ \alpha)(\beta(V))=(\beta \circ \alpha \circ \beta)(V)=(\beta \circ \mathrm{Id})(V)=\beta(V)=E,
$$

and thus $\beta \circ \alpha=\mathrm{Id}$, which finishes the proof.

Below we recall Proposition 8 from [27] which holds over any topological fields with the density property.

Proposition 11.3 Let $X$ be an algebraic $K$-variety and $f$ a rational function on $X$ that is regular on $X^{0} \subset X$. Assume that $\left.f\right|_{X^{0}(K)}$ has a continuous extension $f^{c}: X(K) \rightarrow K$. Let $Z \subset X$ be an irreducible subvariety that is not contained in the singular locus of $X$. Then there is a Zariski dense open subset $Z^{0} \subset Z$ such that $\left.f^{c}\right|_{Z^{0}(K)}$ is a regular function.

We immediately obtain two corollaries:

Corollary 11.4 Let $X$ be an algebraic $K$-variety that is smooth at all $K$-points $X(K)$ and $f$ a rational function on $X$ that is regular on $X^{0} \subset X$. Assume that $\left.f\right|_{X^{0}(K)}$ has a continuous extension $f^{c}: X(K) \rightarrow K$. Then there is a sequence of closed subvarieties

$$
\emptyset=X_{-1} \subset X_{0} \subset \cdots \subset X_{n}=X
$$

such that for $i=0, \ldots, n$ the restriction of $f$ to $X_{i}(K) \backslash X_{i-1}(K)$ is regular. Moreover, we can require that each set $X_{i} \backslash X_{i-1}$ be smooth of pure dimension $i$.

Corollary 11.5 If $f$ is a regulous function on $K^{n}$, then there is a sequence of Zariski closed subsets

$$
\emptyset=E_{-1} \subset E_{0} \subset \cdots \subset E_{n}=K^{n}
$$

such that for $i=0, \ldots, n$ the restriction of $f$ to $E_{i} \backslash E_{i-1}$ is regular. Moreover, we can require that each set $E_{i} \backslash E_{i-1}$ be smooth of pure dimension $i$.

Remark 11.6 Given a finite number of regulous functions $f_{1}, \ldots, f_{p}$, there is a filtration

$$
\emptyset=E_{-1} \subset E_{0} \subset \cdots \subset E_{n}=K^{n}
$$

as in the above corollary such that for $i=0, \ldots, n$ the restriction of each function $f_{j}$, $j=1, \ldots, p$, to $E_{i} \backslash E_{i-1}$ is regular. 
Now, three further consequences of the above corollary will be drawn. We say that a map

$$
f=\left(f_{1}, \ldots, f_{p}\right): K^{n} \rightarrow K^{p}
$$

is $k$-regulous if all its components $f_{1}, \ldots, f_{p}$ are $k$-regulous functions on $K^{n}$.

Corollary 11.7 If two maps

$$
g: K^{m} \rightarrow K^{n} \text { and } f: K^{n} \rightarrow K^{p}
$$

are $k$-regulous, so is its composition $f \circ g$.

Proof Indeed, let $U$ be the common regular locus of the components $g_{1}, \ldots, g_{n}$ of the map $g$ :

$$
U:=\operatorname{dom}\left(g_{1}\right) \cap \ldots \cap \operatorname{dom}\left(g_{n}\right) \subset \mathbb{R}^{m} .
$$

Take a filtration

$$
\emptyset=E_{-1} \subset E_{0} \subset \cdots \subset E_{n}=K^{n}
$$

for the functions $f_{1}, \ldots, f_{p}$ described in Remark 11.6. Then $U$ is the following union of Zariski locally closed subsets

$$
U=\bigcup_{i=0}^{n}\left(U \cap g^{-1}\left(E_{i} \backslash E_{i-1}\right)\right) .
$$

Clearly, one of these sets, say $U \cap g^{-1}\left(E_{i_{0}} \backslash E_{i_{0}-1}\right)$ must be a Zariski dense open subset of $U$ and of $K^{m}$ too. Hence $f \circ g$ is a regular function on $U \cap g^{-1}\left(E_{i_{0}} \backslash E_{i_{0}-1}\right)$, which is the required result.

Corollary 11.8 The zero set $\mathcal{Z}(f)$ of a regulous function $f$ on $K^{n}$ is a closed (in the $K$-topology) constructible subset of $K^{n}$.

Corollary 11.9 The zero set $\mathcal{Z}\left(f_{1}, \ldots, f_{p}\right)$ of finitely many regulous functions $f_{1}, \ldots, f_{p}$ on $K^{n}$ is a closed (in the $K$-topology) constructible subset of $K^{n}$.

Proof This follows directly from Corollary 11.8 and Lemma 10.1.

Hence and by Proposition 11.1, we immediately obtain

Proposition 11.10 The $k$-regulous topology on $K^{n}$ is noetherian.

Corollary 11.11 Every $k$-regulous closed subset of $K^{n}$ is the zero set $\mathcal{Z}(f)$ of a $k$ regulous function $f$ on $K^{n}$ and thus is a closed (in the $K$-topology) constructible subset of $K^{n}$. Hence every $k$-regulous open subset of $K^{n}$ is of the form

$$
\mathcal{D}(f):=K^{n} \backslash \mathcal{Z}(f)=\left\{x \in K^{n}: f(x) \neq 0\right\}
$$


for a $k$-regulous function $f$ on $K^{n}$.

Corollaries 11.11 and 11.7 yield the following

Corollary 11.12 Every k-regulous map $f: K^{n} \rightarrow K^{m}$ is continuous in the $k$ regulous topology.

\section{Regulous Nullstellensatz}

We further assume that the ground field $K$ is a Henselian rank one valued field of equicharacteristic zero and that $K$ is not algebraically closed. Throughout this section, $k$ will be a nonnegative integer. We begin with the following consequence of the Łojasiewicz inequality (Proposition 9.2).

Proposition 12.1 Let $f, g$ be rational functions on $K \mathbb{A}^{n}$ such that $f$ extends to a continuous function on $K^{n}$ and $g$ extends to a continuous function on the $\operatorname{set} \mathcal{D}(f)$. Then the function $f^{s} g$ extends, for $s \gg 0$, by zero through the set $\mathcal{Z}(f)$ to a continuous rational function on $K^{n}$.

Proof We can find a finite composite $\sigma: M \rightarrow K \mathbb{A}^{n}$ of blowups along smooth centers such that the pullbacks

$$
f^{\sigma}:=f \circ \sigma \text { and } g^{\sigma}:=g \circ \sigma
$$

are regular functions at all $K$-points on

$$
M(K) \text { and } M(K) \backslash \sigma^{-1}(\mathcal{Z}(f)),
$$

respectively. Then there are regular functions $p, q$ on $M$ such that

$$
g^{\sigma}=\frac{p}{q} \text { and } \mathcal{Z}(q):=\{y \in M(K): q(y)=0\} \subset \mathcal{Z}\left(f^{\sigma}\right) .
$$

It follows immediately from Proposition 9.2 that the rational function

$$
\frac{\left(f^{\sigma}\right)^{s}}{q}, \text { for } s \gg 0,
$$

extends by zero through the set $\mathcal{Z}\left(f^{\sigma}\right)$ to a continuous function on $M(K)$, whence so does the rational function $\left(f^{\sigma}\right)^{s} \cdot g^{\sigma}$. By the descent property (Corollary 3.6), the continuous function $\left(f^{\sigma}\right)^{s} \cdot g^{\sigma}$ descends to a continuous function on $K^{n}$ that vanishes on $\mathcal{Z}(f)$. This is the required result.

The two corollaries stated below are counterparts of Lemmata 5.1 and 5.2 from [16], established over the real ground field $\mathbb{R}$. 
Corollary 12.2 Let $f$ be a $k$-regulous function on $K^{n}$ and $g$ a $k$-regulous function on the open subset $\mathcal{D}(f)$. Then the function $f^{s} g$, for $s \gg 0$, extends by zero through the zero set $\mathcal{Z}(f)$ to a $k$-regulous function on $K^{n}$. Hence the ring of $k$-regulous functions on $\mathcal{D}(f)$ is the localization $\mathcal{R}^{k}\left(K^{n}\right)_{f}$.

Proof The case $k=0$ is just Proposition 12.1. Now take $s$ large enough so that the partial derivatives

$$
f^{s} \cdot \frac{\partial^{|\alpha|} g}{\partial x^{\alpha}} \text { for } \alpha \in \mathbb{N}^{n},|\alpha|=0,1, \ldots, k
$$

extend to continuous functions on $K^{n}$ vanishing on $\mathcal{Z}(f)$. Then, by Leibniz's rule, $f^{s+k} g$ is a $k$-regulous function on $K^{n}$ and $k$-flat on $\mathcal{Z}(f)$, as desired.

Corollary 12.3 Let $U$ be a $k$-regulous open subset of $K^{n}, f$ a $k$-regulous function on $U$ and $g$ a $k$-regulous function on the open subset $\mathcal{D}(f) \subset U$. Then the function $f^{s} g$ extends, for $s \gg 0$, by zero through the zero set $\mathcal{Z}(f) \subset U$ to a $k$-regulous function on $U$.

Proof By Corollary 11.11, $U=\mathcal{D}(h)$ for a $k$-regulous function on $K^{n}$. From the above corollary, we get

$$
f h^{s} \in \mathcal{R}^{k}\left(K^{n}\right) \text { for } s \gg 0 .
$$

Hence

$$
g \in \mathcal{R}^{k}\left(K^{n}\right)_{f h^{s}}
$$

for integers $s$ large enough, and thus the conclusion follows.

Now we can readily pass to a regulous version of Nullstellensatz, whose proof relies on Corollary 12.2 and the fact that the $k$-regulous topology is noetherian.

Theorem 12.4 If I is an ideal in the ring $\mathcal{R}^{k}\left(K^{n}\right)$ of $k$-regulous functions on $K^{n}$, then

$$
\operatorname{Rad}(I)=\mathcal{I}(\mathcal{Z}(I))
$$

where

$$
\mathcal{I}(E):=\left\{f \in \mathcal{R}^{k}\left(K^{n}\right): f(x)=0 \text { for all } x \in E\right\}
$$

for a subset $E$ of $K^{n}$.

Proof The inclusion $\operatorname{Rad}(I) \subset \mathcal{I}(\mathcal{Z}(I))$ is obvious. For the converse one, apply Corollary 11.11 which says that there is a function $g \in I$ such that $\mathcal{Z}(I)=\mathcal{Z}(g)$. 
Then $\mathcal{Z}(g) \subset \mathcal{Z}(f)$ for any $f \in \mathcal{I}(\mathcal{Z}(I))$, and thus the function $1 / g$ is $k$-regulous on the set $\mathcal{D}(f)$. By Corollary 12.2, we get

$$
\frac{f^{s}}{g} \in \mathcal{R}^{k}\left(K^{n}\right)
$$

for $s \gg 0$ large enough. Hence

$$
f^{s} \in g \cdot \mathcal{R}^{k}\left(K^{n}\right) \subset I,
$$

concluding the proof.

Corollary 12.5 There is a one-to-one correspondence between the radical ideals of the ring $\mathcal{R}^{k}\left(K^{n}\right)$ and the $k$-regulous closed subsets of $K^{n}$. Consequently, the prime ideals of $\mathcal{R}^{k}\left(K^{n}\right)$ correspond to the irreducible $k$-regulous closed subsets of $K^{n}$, and the maximal ideals $\mathfrak{m}$ of $\mathcal{R}^{k}\left(K^{n}\right)$ correspond to the points $x$ of $K^{n}$ so that we get the bijection

$$
K^{n} \ni x \longrightarrow \mathfrak{m}_{x}:=\left\{f \in \mathcal{R}^{k}\left(K^{n}\right): f(x)=0\right\} \in \operatorname{Max}\left(\mathcal{R}^{k}\left(K^{n}\right)\right)
$$

The resulting embedding

$$
\iota: K^{n} \ni x \longrightarrow \mathfrak{m}_{x} \in \operatorname{Spec}\left(\mathcal{R}^{k}\left(K^{n}\right)\right)
$$

is continuous in the $k$-regulous and Zariski topologies. Furthermore, $\iota$ induces a canonical one-to-one correspondence between the $k$-regulous closed subsets of $K^{n}$ and the Zariski closed subsets of Spec $\left(\mathcal{R}^{k}\left(K^{n}\right)\right)$. More precisely, for every $k$-regulous closed subset $V$ of $K^{n}$ there is a unique Zariski closed subset $\widetilde{V}$ of $\mathcal{R}^{k}\left(K^{n}\right)$ such that $V=\iota^{-1}(\widetilde{V})$; actually $\widetilde{V}$ is the Zariski closure of the image $\iota(V)$.

Proof The embedding $\iota$ is continuous by the very definition of the Zariski topology. The last assertion follows immediately from the Nullstellensatz and the fact that the closed subsets of $\operatorname{Spec}\left(\mathcal{R}^{k}\left(K^{n}\right)\right)$ are precisely of the form

$$
\left\{\mathfrak{p} \in \operatorname{Spec}\left(\mathcal{R}^{k}\left(K^{n}\right)\right): \mathfrak{p} \supset I\right\}
$$

where $I$ runs over all radical ideals of $\operatorname{Spec}\left(\mathcal{R}^{k}\left(K^{n}\right)\right)$.

The above corollary along with Proposition 11.10 and Corollary 11.11 yields immediately

Corollary 12.6 With the above notation, the space Spec $\left(\mathcal{R}^{k}\left(K^{n}\right)\right)$ with the Zariski topology is noetherian, and the embedding ı induces a one-to-one correspondence 
between the k-regulous open subsets of $K^{n}$ and the subsets of Spec $\left(\mathcal{R}^{k}\left(K^{n}\right)\right)$ open in the Zariski topology. In particular, every open subset of Spec $\left(\mathcal{R}^{k}\left(K^{n}\right)\right)$ is of the form

$$
\mathcal{U}(f):=\left\{\mathfrak{p} \in \operatorname{Spec}\left(\mathcal{R}^{k}\left(K^{n}\right)\right): f \notin \mathfrak{p}\right\}, \quad f \in \mathcal{R}^{k}\left(K^{n}\right),
$$

corresponding to the subset $\mathcal{D}(f)$ of $K^{n}$.

Remark 12.7 As demonstrated in [16] (see also [33, Ex. 6.11]), the $\operatorname{ring} \mathcal{R}^{k}\left(K^{n}\right)$ is not noetherian for all $k, n \in \mathbb{N}, n \geq 2$.

From Theorem 12.4 and Corollary 11.11, we immediately obtain

Corollary 12.8 Every radical ideal of $\mathcal{R}^{k}\left(K^{n}\right)$ is the radical of a principal ideal of $\mathcal{R}^{k}\left(K^{n}\right)$.

Finally, we return to the comparison of the regulous and constructible topologies. Below we state the non-archimedean version of [16, Theorem 6.4] by FichouHuisman-Mangolte-Monnier, which says that those topologies coincide in the real algebraic geometry. The proof relies on their Lemmata 5.1 and 5.2, and it can be repeated verbatim in the case of the ground fields $K$ studied in our paper by means of Corollaries 12.2 and 12.3.

Proposition 12.9 The $k$-regulous closed subsets of $K^{n}$ are precisely the closed (in the $K$-topology) constructible subsets of $K^{n}$.

The above theorem along with Corollary 11.2 and Corollary 12.5 yields the following

Corollary 12.10 There are one-to-one correspondences between the prime ideals of the ring $\mathcal{R}^{k}\left(K^{n}\right)$, the irreducible closed constructible subsets of $K^{n}$ and the irreducible Zariski closed subsets of $K^{n}$.

Corollary 12.11 The dimension of the topological space $K^{n}$ with the regulous topology and the Krull dimension of the ring $\mathcal{R}^{k}\left(K^{n}\right)$ is $n$.

\section{Quasi-coherent regulous sheaves}

The concepts of quasi-coherent $k$-regulous sheaves on $K^{n}$ and $k$-regulous affine varieties, $k \in \mathbb{N} \cup\{\infty\}$, can be introduced over valued fields studied in this paper, similarly as by Fichou-Huisman-Mangolte-Monnier [16] over the real ground field $\mathbb{R}$. Also, the majority of their results concerning these concepts carry over to the non-archimedean geometry with similar proofs. For the sake of completeness, we provide an exposition of the theory of quasi-coherent regulous sheaves. Here we shall deal only with $k$-regulous functions with a nonnegative integer $k$, because for $k=\infty$ we encounter the classical case of regular functions and quasi-coherent algebraic sheaves.

Consider an affine scheme $Y=\operatorname{Spec}(A)$ with structure sheaf $\mathcal{O}_{Y}$. Any $A$-module $M$ determines a quasi-coherent sheaf $\widetilde{M}$ on $Y$ (cf. [21, Chap. II]). The functor $M \longmapsto$ 
$\widetilde{M}$ gives an equivalence of categories between the category of $A$-modules and the category of quasi-coherent $\mathcal{O}_{Y}$-modules. Its inverse is the global sections functor

$$
\mathcal{F} \longmapsto H^{0}(Y, \mathcal{F})
$$

(cf. [21, Chap. II, Corollary 5.5]).

Denote by $\widetilde{\mathcal{R}}^{k}$ the structure sheaf of the affine scheme $\operatorname{Spec}\left(\mathcal{R}^{k}\left(K^{n}\right)\right)$ and by $\mathcal{R}^{k}$ the sheaf of $k$-regulous function germs (in the $k$-regulous topology equal to the constructible topology) on $K^{n}$. It follows directly from Corollaries 12.5 and 12.2 that the restriction $\iota^{-1} \widetilde{\mathcal{R}}^{k}$ of $\widetilde{\mathcal{R}}^{k}$ to $K^{n}$ coincides with the sheaf $\mathcal{R}^{k}$; conversely, $\iota_{*} \mathcal{R}^{k}=$ $\widetilde{\mathcal{R}}^{k}$.

By a $k$-regulous sheaf $\mathcal{F}$ we mean a sheaf of $\mathcal{R}^{k}$-modules. Again, it follows immediately from Corollaries 12.5 and 12.6 that the functor $\iota^{-1}$ of restriction to $K^{n}$ gives an equivalence of categories between $\widetilde{\mathcal{R}}^{k}$-modules and $\mathcal{R}^{k}$-modules. Its inverse is the direct image functor $\iota_{*}$.

We say that $\mathcal{F}$ is a quasi-coherent $k$-regulous sheaf on $K^{n}$ if it is the restriction to $K^{n}$ of a quasi-coherent $\widetilde{\mathcal{R}}^{k}$-module. Thus the functor $\iota^{-1}$ induces an equivalence of categories between quasi-coherent $\widetilde{\mathcal{R}}^{k}$-modules and quasi-coherent $\mathcal{R}^{k}$-modules, whose inverse is the direct image functor $\iota_{*}$. For any $\mathcal{R}^{k}\left(K^{n}\right)$-module $M$, we shall denote by $\widetilde{M}$ both the associated sheaf on $\operatorname{Spec}\left(\mathcal{R}^{k}\left(K^{n}\right)\right)$ and its restriction to $K^{n}$. This abuse of notation does not lead to confusion. We thus obtain the following version of Cartan's theorem A:

Theorem 13.1 The functor $M \longmapsto \tilde{M}$ gives an equivalence of categories between the category of $\mathcal{R}^{k}\left(K^{n}\right)$-modules and the category of quasi-coherent $\mathcal{R}^{k}$-modules. Its inverse is the global sections functor

$$
\mathcal{F} \longmapsto H^{0}\left(K^{n}, \mathcal{F}\right) .
$$

In particular, every quasi-coherent sheaf $\mathcal{F}$ is generated by its global sections $H^{0}\left(K^{n}, \mathcal{F}\right)$.

The regulous version of Cartan's theorem B, stated below, follows directly from the version for affine (not necessarily noetherian in view of Remark 12.7) schemes (cf. [20, Theorem 1.3.1]) via the discussed equivalence of categories (being the functor $\iota^{-1}$ of restriction to $K^{n}$ ).

Theorem 13.2 If $\mathcal{F}$ is a quasi-coherent $k$-regulous sheaf on $K^{n}$, then

$$
H^{i}\left(K^{n}, \mathcal{F}\right)=0 \text { for all } i>0 .
$$

Corollary 13.3 The global sections functor

$$
\mathcal{F} \longmapsto H^{0}\left(K^{n}, \mathcal{F}\right)
$$

on the category of quasi-coherent $k$-regulous sheaves on $K^{n}$ is exact. 
Let $V$ be a $k$-regulous closed subset of $K^{n}$ and $\Im(V)$ the sheaf of those $k$-regulous function germs on $K^{n}$ that vanish on $V$. It is a quasi-coherent sheaf of ideals of $\mathcal{R}^{k}$, the sheaf $\mathcal{R}^{k} / \mathfrak{I}(V)$ has support $V$ and is generated by its global sections (Theorem A); moreover

$$
H^{0}\left(K^{n}, \mathcal{R}^{k} / \Im(V)\right)=H^{0}\left(K^{n}, \mathcal{R}^{k}\right) / H^{0}\left(K^{n}, \mathfrak{I}(V)\right)
$$

(Theorem B). The subset $V$ inherits the $k$-regulous topology from $K^{n}$ and constitutes, together with the restriction $\mathcal{R}_{V}^{k}$ of the sheaf $\mathcal{R}^{k} / \mathfrak{I}(V)$ to $V$, a locally ringed space of $K$-algebras, called an affine $k$-regulous subvariety of $K^{n}$. More generally, by an affine $k$-regulous variety we mean any locally ringed space of $K$-algebras that is isomorphic to an affine $k$-regulous subvariety of $K^{n}$ for some $n \in \mathbb{N}$.

We can define in the ordinary fashion the category of quasi-coherent $\mathcal{R}_{V}^{k}$-modules. Each such module extends trivially by zero to a quasi-coherent $\mathcal{R}^{k}$-module on $K^{n}$. The sections $\mathcal{R}_{V}^{k}(V)$ of the structure sheaf $\mathcal{R}_{V}^{k}$ are called $k$-regulous functions on $V$. It follows from Cartan's theorem B that each $k$-regulous function on $V$ is the restriction to $V$ of a $k$-regulous function on $K^{n}$. Hence we immediately obtain the following two results.

Proposition 13.4 Let $W$ and $V$ be two affine $k$-regulous subvarieties of $K^{m}$ and $K^{n}$, respectively. Then the following three conditions are equivalent:

1) $f: W \rightarrow V$ is a morphism of locally ringed spaces;

2) $f=\left(f_{1}, \ldots, f_{n}\right): W \rightarrow K^{n}$ where $f_{1}, \ldots, f_{n}$ are $k$-regulous functions on $W$ such that $f(W) \subset V$;

3) $f$ extends to a $k$-regulous map $K^{m} \rightarrow K^{n}$.

We then call $f: W \rightarrow V$ a $k$-regulous map.

Corollary 13.5 Let $W, V$ and $X$ be affine $k$-regulous subvarieties of $K^{m}, K^{n}$ and $K^{p}$, respectively. If two maps

$$
g: W \rightarrow V \text { and } f: V \rightarrow X
$$

are k-regulous, so is its composition $f \circ g$.

It is clear that Cartan's theorems remain valid for quasi-coherent $k$-regulous sheaves on affine $k$-regulous varieties $V$.

Corollary 13.6 The functor $M \longmapsto \widetilde{M}$ gives an equivalence of categories between the category of $\mathcal{R}_{V}^{k}(V)$-modules and the category of quasi-coherent $\mathcal{R}_{V}^{k}$-modules. Its inverse is the global sections functor

$$
\mathcal{F} \longmapsto H^{0}(V, \mathcal{F})
$$

In particular, every quasi-coherent sheaf $\mathcal{F}$ is generated by its global sections $H^{0}(V, \mathcal{F})$. 
Corollary 13.7 If $\mathcal{F}$ is a quasi-coherent $k$-regulous sheaf on $V$, then

$$
H^{i}(V, \mathcal{F})=0 \text { for all } i>0 \text {. }
$$

Corollary 13.8 The global sections functor

$$
\mathcal{F} \longmapsto H^{0}(V, \mathcal{F})
$$

on the category of quasi-coherent $k$-regulous sheaves on $V$ is exact.

Note that every non-empty $k$-regulous open subset $U$ of $K^{n}$ is an affine $k$-regulous variety. Indeed, if $U=\mathcal{D}(f)$ for a $k$-regulous function $f$ on $K^{n}$ (Corollary 11.11), then $U$ is isomorphic to the affine $k$-regulous subvariety

$$
V:=\mathcal{Z}(y f(x)-1) \subset K_{x}^{n} \times K_{y}
$$

Remark 13.9 Consider a smooth algebraic subvariety $X$ of the affine space $K \mathbb{A}^{n}$. We may look at the set $V:=X(K)$ of its $K$-points both as an algebraic variety $X(K)$ and as a $k$-regulous subvariety $V$ of $K^{n}$. Every function $f: V \rightarrow K$ that is $k$-regulous on $V$ in the second sense remains, of course, $k$-regulous on $X(K)$ in the sense of the definition from the beginning of Sect. 11.

Open problem. The problem whether the converse implication is true for $k>0$ is unsolved as yet.

For $k=0$ the answer is in the affirmative and follows immediately from Theorem 10.2. Indeed, every continuous hereditarily rational function $f$ on $X(K)$ extends to a continuous rational function on $K^{n}$, whence $f$ is regulous on $V$. This theorem was proven for real and $p$-adic varieties in [27].

Finally, we wish to give a criterion for a continuous function to be regulous. It relies on Theorem 10.2 on extending continuous hereditarily rational functions on algebraic $K$-varieties.

Proposition 13.10 Let $V$ be an affine regulous subvariety of $K^{n}$ and $f: V \rightarrow K a$ function continuous in the $K$-topology. Then a necessary and sufficient condition for $f$ to be a regulous function is the following:

(*) For every Zariski closed subset $Z$ of $K^{n}$, there exist a Zariski dense open subset $U$ of the Zariski closure of $V \cap Z$ in $K^{n}$ and a regular function $g$ on $U$ such that

$$
f(x)=g(x) \text { for all } x \in V \cap Z \cap U \text {. }
$$

Proof By Corollary 11.5, the necessary condition is clear, because $f$ is the restriction to $V$ of a regulous function on $K^{n}$ (Corollary 13.3 to Cartan's theorem B).

In order to prove the sufficient condition, we proceed with induction with respect to the dimension $d$ of the set $V$ which is a closed (in the $K$-topology) constructible subset of $K^{n}$. The case $d=0$ is trivial. Assuming the assertion to hold for dimensions 
less than $d$, we shall prove it for $d$. So suppose $V$ is of dimension $d$. By Corollary 11.2, the Zariski closure $W$ of $V$ in $K^{n}$ is of dimension $d$ and we have

$$
W^{0}:=\{x \in W: W \text { is smooth of dimension } d \text { at } x\} \subset V
$$

Obviously, $W^{*}:=W \backslash W^{0}$ is a Zariski closed subset of $K^{n}$ of dimension less than $d$. Therefore, $Y:=V \cap W^{*}$ is a regulous closed subset of $V$ of dimension less than $d$ and

$$
W=W^{0} \cup W^{*} \subset V \cup W^{*} \subset W
$$

Since the restriction $f \mid Y$ satisfies condition (*), it is a regulous function on $Y$ by the induction hypothesis. It is thus the restriction to $Y$ of a regulous function $F$ on $K^{n}$ (Corollary 13.3 to Cartan's theorem B).

Further, the function $f$ and the restriction $F \mid W^{*}$ can be glued to a function

$$
g: W=V \cup W^{*} \rightarrow K, \quad g(x)=\left\{\begin{array}{l}
f(x): x \in V \\
F(x): x \in W^{*}
\end{array}\right.
$$

which satisfies condition (*) as well. Now, it follows from Theorem 10.2 that $g$ extends to a regulous function $G$ on $K^{n}$. Since $f$ is the restriction to $V$ of the function $G$ which is regulous, so is $f$, as required.

Acknowledgements The author wishes to express his gratitude to János Kollár and Wojciech Kucharz for several stimulating discussions on the topics of this paper.

Open Access This article is distributed under the terms of the Creative Commons Attribution 4.0 International License (http://creativecommons.org/licenses/by/4.0/), which permits unrestricted use, distribution, and reproduction in any medium, provided you give appropriate credit to the original author(s) and the source, provide a link to the Creative Commons license, and indicate if changes were made.

\section{References}

1. Andreotti, A., Bombieri, E.: Sugli omeomorfismi delle varietà algebriche. Ann. Scuola Norm. Sup Pisa 23(3), 431-450 (1969)

2. Andreotti, A., Norguet, F.: La convexité holomorphe dans l'espace analytique des cycles d'une variété algébrique. Ann. Scuola Norm. Sup. Pisa 21(3), 31-82 (1967)

3. Berkovich, V.: Spectral Theory and Analytic Geometry over Non-Archimedean Fields, Math. Surveys and Monographs. AMS, Providence (1990)

4. Besarab, S.: Relative elimination of quantifiers for Henselian valued fields. Ann. Pure Appl. Logic 53, 51-74 (1991)

5. Bochnak, J., Coste, M., Roy, M.-F.: Real Algebraic Geometry, Ergebnisse der Mathematik und ihrer Grenzgebiete. Springer, Berlin (1998)

6. Bosch, S., Güntzer, U., Remmert, R.: Non-Archimedian Analysis: A Systematic Approach to Rigid Analytic Geometry, Grundlehren der math. Wiss. Springer, Berlin (1984)

7. Bourbaki, N.: Algèbre Commutative. Hermann, Paris (1962)

8. Bruhat, F., Cartan, H.: Sur la structure des sous-ensembles analytiques réels. C. R. Acad. Sci. 244, 988-990 (1957)

9. Cherlin, G.: Model Theoretic Algebra, Selected Topics, Lect. Notes Math., vol. 521. Springer, Berlin (1976) 
10. Cluckers, R., Loeser, F.: b-Minimality. J. Math. Logic 7(2), 195-227 (2007)

11. Cluckers, R., Lipshitz, L.: Fields with analytic structure. J. Eur. Math. Soc. 13, 1147-1223 (2011)

12. Cluckers, R., Lipshitz, L.: Strictly convergent analytic structures. J. Eur. Math. Soc. arXiv:1312.5932 [math.LO] (2015)

13. Denef, J.: p-Adic semi-algebraic sets and cell decomposition. J. Rein. Angew. Math. 369, 154-166 (1986)

14. Denef, J., van den Dries, L.: p-Adic and real subanalytic sets. Ann. Math. 128, 79-138 (1988)

15. Engler, A.J., Prestel, A.: Valued Fields. Springer, Berlin (2005)

16. Fichou, G., Huisman, J., Mangolte, F., Monnier, J.-P.: Fonctions régulues, J. Rein. Angew. Math. (2015). doi:10.1515/crelle-2014-0034

17. Fisher, B.: A note on Hensel's lemma in several variables. Proc. Am. Math. Soc. 125(11), 3185-3189 (1997)

18. Forey, A.: Motivic local density. arXiv:1512.00420 [math.LO] (2015)

19. Grauert, H., Remmert, R.: Analytische Stellenalgebren, Grundlehren der math. Wiss. Springer, Berlin (1971)

20. Grothendieck, A.: Éléments de Géométríe Algébrique. III. Étude cohomologique des faisceaux cohérents, Publ. Math. IHES 11 (1961) and 17 (1963)

21. Hartshorne, R.: Algebraic Geometry, Graduate Texts in Mathematics. Springer, Berlin (1977)

22. Hrushovski, E., Kazhdan, D.: Integration in valued fields. In: Algebraic Geometry and Number Theory, Progr. Math., vol. 253, pp. 261-405. Birkhäuser Boston, Boston, MMA (2006)

23. Hrushovski, E., Loeser, F.: Non-Archimedean Tame Topology and Stably Dominated Types, Annals of Mathematics Studies. Princeton University Press, Princeton (2016)

24. Kaplansky, I.: Maximal fields with valuations I and II. Duke Math. J. 9 (1942), 303-321 and 12 (1945), 243-248

25. Kollár, J.: Lectures on Resolution of Singularities, Ann. Math. Studies. Princeton University Press, Princeton (2007)

26. Kollár, J.: Singularities of the minimal model program, (With a collaboration of S. Kovács) Cambridge Tracts in Mathematics. Cambridge University Press, Cambridge (2013)

27. Kollár, J., Nowak, K.: Continuous rational functions on real and $p$-adic varieties. Math. Zeitschrift 279, 85-97 (2015)

28. Kucharz, W.: Continuous rational maps into the unit 2-sphere. Arch. Math. 102, 257-261 (2014)

29. Kucharz, W.: Approximation by continuous rational maps into spheres. J. Eur. Math. Soc. 16, 15551569 (2014)

30. Kucharz, W.: Continuous rational maps into spheres. Math. Zeitschrift. (2016). doi:10.1007/ s00209-016-1639-4

31. Kucharz, W., Kurdyka, K.: Stratified-algebraic vector bundles. J. Rein. Angew. Math. (2016). doi:10. 1515/crelle-2015-0105

32. Kunz, E.: Introduction to Commutative Algebra and Algebraic Geometry. Birkhäuser, Boston (1985)

33. Kurdyka, K.: Ensemble semi-algébriques symétriques par arcs. Math. Ann. 282, 445-462 (1988)

34. Lipshitz, L., Robinson, Z.: Uniform properties of rigid subanalytic sets. Trans. Am. Math. Soc. 357(11), 4349-4377 (2005)

35. Łojasiewicz, S.: Ensembles Semi-Analytiques. I.H.E.S, Bures-sur-Yvette (1965)

36. Milnor, J.: Singular Points of Complex Hypersurfaces. Princeton University Press, Princeton (1968)

37. Nowak, K.J.: On the Euler characteristic of the links of a set determined by smooth definable functions. Ann. Pol. Math. 93, 231-246 (2008)

38. Nowak, K.J.: Supplement to the paper "Quasianalytic perturbation of multiparameter hyperbolic polynomials and symmetric matrices" (Ann. Polon. Math. 101 (2011) 275-291). Ann. Polon. Math. 103(2012), 101-107

39. Nowak, K.J.: Some results from algebraic geometry over complete discretely valued fields. arXiv:1311.2051 [math.AG] (2013)

40. Nowak, K.J.: Some results from algebraic geometry over Henselian real valued fields. arXiv:1312.2935 [math.AG] (2013)

41. Pas, J.: Uniform p-adic cell decomposition and local zeta functions. J. Rein. Angew. Math. 399, 137172 (1989)

42. Pas, J.: On the angular component map modulo p. J. Symb. Logic 55, 1125-1129 (1990)

43. Robinson, A.: Complete Theories. North-Holland, Amsterdam (1956) 
44. Scowcroft, P., van den Dries, L.: On the structure of semi-algebraic sets over $p$-adic fields. J. Symb. Logic 53(4), 1138-1164 (1988)

45. Wallace, A.H.: Algebraic approximation of curves. Can. J. Math. 10, 242-278 (1958)

46. Zariski, O., Samuel, P.: Commutative Algebra, vol. II. Van Nostrand, Princeton (1960) 